\title{
GRILLIX: A 3D turbulence code based on the flux-coordinate independent approach
}

\author{
Andreas Stegmeir ${ }^{\mathrm{a}}$, David Coster ${ }^{\mathrm{a}}$, Alexander Ross $^{\mathrm{a}}$, Omar Maj $^{\mathrm{a}}$, Karl Lackner ${ }^{\mathrm{a}}$, Emanuele Poli $^{\mathrm{a}}$ \\ ${ }^{a}$ Max-Planck-Institut für Plasmaphysik, D-85748 Garching, Germany
}

\begin{abstract}
The GRILLIX code is presented with which plasma turbulence/transport in various geometries can be simulated in 3D. The distinguishing feature of the code is that it is based on the flux-coordinate independent approach (FCI) [1, 2]. Cylindrical or Cartesian grids are used on which perpendicular operators are discretised via standard finite difference methods and parallel operators via a field line tracing and interpolation procedure (field line map). This offers a very high flexibility with respect to geometry, especially a separatrix with X-point(s) or a magnetic axis can be treated easily in contrast to approaches which are based on field aligned coordinates and suffer from coordinate singularities. Aiming finally for simulation of edge and scrape-off layer turbulence, an isothermal electrostatic drift-reduced Braginskii model [3] has been implemented in GRILLIX. We present the numerical approach, which is based on a toroidally staggered formulation of the FCI, we show verification of the code with the method of manufactured solutions (MMS) and show a benchmark based on a TORPEX blob experiment, previously performed by several edge/SOL codes [4]. Examples for slab, circular, limiter and diverted geometry are presented. Finally, the results show that the FCI approach in general and GRILLIX in particular are viable approaches in order to tackle simulation of edge/SOL turbulence in diverted geometry.
\end{abstract}

Keywords: Flux-coordinate independent (FCI), X-point, separatrix, turbulence, edge, scrape-off layer (SOL), Method of Manufactured Solutions (MMS)

\section{Introduction}

The edge and scrape-off layer (SOL) of magnetic fusion devices play a crucial role for the achievement of a sustainable fusion energy source. Many core parameters are often largely set by edge/SOL conditions [5, 6] and especially the problem of heat exhaust is one of the major challenges for large tokamaks like ITER or DEMO [7]. However, the modelling of this region exhibits many difficulties, and several code projects, like HESEL [8], GBS [9, 10], HERMES (BOUT++) [11] and TOKAM3X [12], are therefore devoted to simulation and prediction of turbulent transport in the edge/SOL region. A particular challenge at modelling of the edge/SOL arises due to its complex geometry. Tokamaks are nowadays mostly based on the divertor concept, where in between the edge with toroidally nested flux surfaces and the SOL with open magnetic field lines intersecting divertor plates, there is a magnetic separatrix with $\mathrm{X}$-point. In order to exploit the fact that turbulent structures are usually strongly elongated along magnetic field lines, it would be very convenient from the theoretical, from the numerical and from the computational point of view to employ field/flux aligned coordinates [13]. However, field/flux aligned coordinates become singular on the separatrix/X-point, which justifies and motivates the use of a flux-coordinate independent approach.

In the flux-coordinate independent approach (FCI) $[1,2,14]$ the simulation domain of e.g. a tokamak is spanned with a cylindrical grid, which is toroidally sparse in order to exploit the

Email address: Andreas.Stegmeir@ipp.mpg.de (Andreas Stegmeir) flute mode character of structures $\left(k_{\perp} \gg k_{\|}\right)$. The discretisation of perpendicular (w.r.t. magnetic field) operators is straight forward as their stencil remains within Cartesian poloidal planes, and parallel operators are discretised according to a finite difference along magnetic field lines by means of a field line tracing and interpolation procedure (field line map). The basic feasibility of the FCI was shown recently mainly with the codes FENICIA $[1,15,16]$ and GRILLIX during its development. Solutions to critical numerical issues, like numerical perpendicular diffusion, map distortion and the treatment of boundary conditions were found and are discussed in $[1,2,15,17,18,19,20]$.

As a proof of concept the FCI has been adopted in the past only to pure advection/diffusion problems and simple models like drift wave turbulence and ion temperature gradient driven turbulence in magnetic island geometry [1, 21, 16, 22, 23]. In this paper we present for the first time the application of the FCI to a physically relevant fluid model for the edge/SOL, i.e. a drift reduced Braginskii model [3, 24] with sheath boundary conditions at the target plates. This model is implemented in the MPI+OpenMP parallelised code GRILLIX. In section 2 we present the physical model with the employed boundary conditions and discuss its conservation properties. In section 3 we present the numerical approach in detail, i.e. how the model is discretised within the FCI approach and how the boundary conditions are treated, a subtle but critical issue. Details on implementation and parallelisation are given, too. After this follows section 4 on verification and validation of GRILLIX. We employ the Method of Manufactured Solutions (MMS) [25] for verification of the implementation, and validate against an experiment at the TORPEX device, an exercise that had been 
performed previously by many other edge/SOL codes [4]. In order to show the high geometric flexibility of GRILLIX we give examples in section 5 in various magnetic configurations. Concluding statements and an outlook are given in section 6.

\section{Physical model}

\subsection{Equations}

Firstly, we assume that the conditions for a fluid description of the plasma, like the Braginskii equations [24], are satisfied, i.e. the mean free path is short against the characteristic scale length of the system $\left(\lambda_{m f p} \ll R_{0}\right)$. This assumption is usually only fulfilled at low temperatures, and might therefore become especially in the edge region questionable. Secondly, we apply the drift reduction based on the assumption that frequencies of interest are small compared to the ion-gyro frequency $\omega \ll \Omega_{i}$, yielding the model described in [3].

Further simplifying assumptions are isothermal electrons $\left(T_{e}=\right.$ const. $)$, cold ions $\left(T_{i} / T_{e} \ll 1\right)$, no electron inertia $\left(m_{e} / m_{i} \ll 1\right)$ and neglect of magnetic induction $\left(\beta_{e}:=4 \pi p_{e} / B^{2} \ll 1\right)$. Moreover we will apply the Boussinesq approximation in the vorticity equation and neglect the polarisation velocity in the advective derivative. The latter approximations are commonly made in the community $[8,11,12]$ and have subtle effects on the energy theorem, which is clarified in section 2.2. Although these assumptions might appear too strong for typical edge/SOL conditions, the resulting model retains a physical relevance for the edge/SOL as it still contains the main driving instabilities, i.e. drift-waves and ballooning modes. Moreover, as discussed in more detail in the outlook (section 6), an extension of the code which relaxes these assumptions does not pose any problems of principle to the code or the FCI approach in general and will be pursued in the future.

For purpose of normalisation we introduce a reference density $n_{0}$, a reference magnetic field $B_{0}$, a reference drift scale $\rho_{s}:=c \sqrt{T_{e} m_{i}} /\left(e B_{0}\right)$ and a parallel scale length $R_{0}$, being typically the major radius. This fixes the dimensionless parameter $\delta:=R_{0} / \rho_{s}$. Perpendicular scales are normalised with respect to $\rho_{s}$, parallel scales with respect to $R_{0}$ and time is measured in units of $R_{0} / c_{s}$ with the sound speed $c_{s}=\sqrt{T_{e} / m_{i}}$. Velocities are normalized with respect to the sound speed and the electrostatic potential $\phi$ against $e / T_{e}$.

Under the given assumptions the velocity of the ion/electron fluid is described via a perpendicular drift motion and a parallel streaming:

$$
\begin{aligned}
\mathbf{u}^{i} & =\mathbf{u}_{E}+\mathbf{u}_{p o l}+u_{\|}^{i} \mathbf{b} . \\
\mathbf{u}^{e} & =\mathbf{u}_{E}+\mathbf{u}_{*}+\left(u_{\|}^{i}-\frac{j_{\|}}{n}\right) \mathbf{b}
\end{aligned}
$$

with $\mathbf{b}:=\mathbf{B} / B$ the unit vector of the magnetic field and $j_{\|}$the parallel current. The normalised $E \times B$ drift velocity $\mathbf{u}_{E}$, the electron diamagnetic drift velocity $\mathbf{u}_{*}$ and the polarisation drift $\mathbf{u}_{p o l}$ are defined respectively as:

$\mathbf{u}_{E}:=\frac{1}{B^{2}} \mathbf{B} \times \nabla \phi, \quad \mathbf{u}_{*}:=-\frac{1}{n B^{2}} \mathbf{B} \times \nabla n, \quad \mathbf{u}_{p o l}:=\delta^{-1} \frac{\mathbf{B}}{B^{2}} \times \frac{d^{i}}{d t} \mathbf{u}_{E}$, with the ion advective derivative:

$$
\frac{d^{i}}{d t}:=\frac{\partial}{\partial t}+\left[\delta \mathbf{u}_{E}+u_{\|}^{i} \mathbf{b}\right] \cdot \nabla
$$

We note that we have neglected here the advection with the polarisation velocity, and the effect of this on the energy theorem is clarified in section 2.2. Effects arising from magnetic inhomogeneity are respected and enter the model via the curvature operator:

$$
C(f):=-\delta \nabla \cdot\left(\frac{B}{B^{2}} \times \nabla f\right)
$$

and we define the following operators in order to add perpendicular/parallel dissipation:

$$
\begin{aligned}
\mathcal{D}_{\perp}(f) & :=\nabla \cdot\left[v_{f} \nabla_{\perp}\left(\nabla_{\perp}^{2 N} f\right)\right], \\
\mathcal{D}_{\|}(f) & :=\nabla \cdot\left(\mu_{f} \mathbf{b} \nabla_{\|} f\right),
\end{aligned}
$$

i.e. for $N>0$ hyper viscosity is applied in the perpendicular direction. Dissipation is mainly needed for numerical reasons, or the dissipation coefficients $v_{f}, \mu_{f}$ can also be chosen such that GRILLIX is run as transport code.

Finally, the physical model consists of the continuity equation:

$$
\begin{aligned}
\frac{\partial}{\partial t} n+ & \delta \mathbf{u}_{E} \cdot \nabla n+\nabla \cdot\left(n u_{\|}^{i} \mathbf{b}\right)=-C(n)+n C(\phi)+\nabla \cdot\left(\mathbf{b} j_{\|}\right) \\
& +S_{n}+\mathcal{D}_{\perp}(n)+\mathcal{D}_{\|}(n)
\end{aligned}
$$

where we have added a particle source $S_{n}$. The equation for the ion parallel velocity is:

$$
\frac{d^{i}}{d t} u_{\|}^{i}=-\nabla_{\|} \log n-\frac{S_{n} u_{\|}^{i}}{n}+\mathcal{D}_{\perp}\left(u_{\|}^{i}\right)+\mathcal{D}_{\|}\left(u_{\|}^{i}\right)
$$

and Ohm's law:

$$
j_{\|}=\sigma \nabla_{\|}(\log n-\phi),
$$

with $\sigma:=\frac{c_{s}}{R_{0}} \frac{m_{i}}{m_{e}} \frac{1}{0.51 v_{e}}$ the dimensionless conductivity. The quasi-neutrality condition $\nabla \cdot\left(n \mathbf{u}^{i}-n \mathbf{u}^{e}\right)=0$ yields (w/o dissipation):

$$
\nabla \cdot\left(\frac{n}{B^{2}} \frac{d^{i}}{d t} \nabla_{\perp} \phi\right)=\nabla \cdot\left(\mathbf{b} j_{\|}\right)-C(n),
$$

to which we apply the Boussinesq approximation. The Boussinesq approximation modifies the energy theorem and an energetic consistent form is [11]:

$$
\nabla \cdot\left(\frac{n}{B^{2}} \frac{d^{i}}{d t} \nabla_{\perp} \phi\right) \rightarrow \frac{\partial}{\partial t} \Omega+\nabla \cdot\left(\delta \mathbf{u}_{E} \Omega\right),
$$

with the vorticity $\Omega:=\nabla \cdot\left(\nabla_{\perp} \phi / B^{2}\right)$. The Boussinesq approximation is routinely applied in the community since it simplifies the computational treatment significantly (see section 3.5), but a priori there is no justification for it as large fluctuations are generally present in the edge/SOL [26]. A multigrid solver is currently under development in order to relax the Boussinesq approximation within GRILLIX. Finally the vorticity equation implemented in GRILLIX is:

$$
\frac{\partial}{\partial t} \Omega+\delta \mathbf{u}_{E} \cdot \nabla \Omega=\nabla \cdot\left(\mathbf{b} j_{\|}\right)+\Omega C(\phi)-C(n)+\mathcal{D}_{\perp}(\Omega) .
$$




\subsection{Energy theorem}

The perpendicular respectively parallel kinetic energy densities are defined as:

$$
\epsilon_{\perp}:=\frac{1}{2} \mathbf{u}_{E}^{2}=\frac{\left(\nabla_{\perp} \phi\right)^{2}}{2 B^{2}}, \quad \epsilon_{\|}:=\frac{1}{2} n u_{\|}^{i 2} .
$$

Note that the density does not enter the definition of the perpendicular kinetic energy due to the Boussinesq approximation. The time-evolution of the energy densities are easily obtained as:

$$
\begin{aligned}
& \frac{\partial}{\partial t} \epsilon_{\perp}+\nabla \cdot\left[\phi \mathbf{j}+n \mathbf{u}_{E}\right]=j_{\|} \nabla_{\|} \phi+n \nabla \cdot \mathbf{u}_{E}+D_{\epsilon_{\perp}} \\
& \frac{\partial}{\partial t} \epsilon_{\|}+\nabla \cdot\left[\epsilon_{\|}\left(\delta \mathbf{u}_{E}+u_{\|}^{i 2} \mathbf{b}+\frac{\mathbf{u}_{p o l}}{n}\right)\right]-\mathbf{u}_{p o l} \cdot \nabla\left(\frac{\epsilon_{\|}}{n}\right)=-u_{\|}^{i} \nabla_{\|} n+D_{\epsilon_{\|}},
\end{aligned}
$$

where the term under the divergence are transport terms. In $D_{\epsilon_{\perp}}$ and $D_{\epsilon_{\|}}$terms arising from dissipation and sources are summarized, for which explicit expressions can be found in Appendix A. The other terms on the right hand side would represent transfer terms to the magnetic respectively thermal energy within a more complete model $[3,27]$, i.e. in our electrostatic/isothermal model these terms appear as sources/sinks with a well defined physical interpretation. The division of the parallel kinetic energy by $n$ in terms with $\mathbf{u}_{p o l}$ is a consequence of the Boussinesq approximation. Finally, the term $\mathbf{u}_{\text {pol }} \cdot \nabla\left(\epsilon_{\|} / n\right)$ is a spurious term, which arises since we have neglected the polarisation velocity in the ion advective derivative. However, this term is expected to be small as the polarisation velocity is of higher order.

\subsection{Boundary conditions}

In general the simulation domain in GRILLIX is limited by an outer flux surface $\psi_{\max }$ and in order to save computational resources optionally by an inner magnetic flux surface $\psi_{\text {min }}$. If the outer limiting flux surface is outside the separatrix $\left(\psi_{\max }>\right.$ $\psi_{x}$ ) additional boundaries at the divertor or limiter plates are present (see fig. 1).

We want to avoid fluxes of particles and energy through the inner limiting flux surface if present $[11,28]$, which implies according to equations (15) and (16) that $\mathbf{u}_{E}$ must be tangential to flux surfaces at the boundary.

$$
\begin{aligned}
\left.\phi\right|_{\psi_{\text {min }}} & =0, \\
\left.\mathbf{e}_{\psi} \cdot \nabla n\right|_{\psi_{\text {min }}} & =0, \\
\left.\mathbf{e}_{\psi} \cdot \nabla u_{\|}^{i}\right|_{\psi_{\text {min }}} & =0, \\
\left.\Omega\right|_{\psi_{\text {min }}} & =0,
\end{aligned}
$$

The same conditions are applied at the outer limiting flux surface if open magnetic field lines are present. For simulations with purely closed magnetic field line, i.e. $\psi_{\max }<\psi_{x}$ a particle sink is introduced via:

$$
\left.n\right|_{\psi_{\max }<\psi_{x}}=C
$$

with a constant $C>0$. Additionally buffer zones close to the limiting flux surfaces are present, where a strong perpendicular diffusion to all quantities is applied (see section 5).

At the target plates sheath boundary conditions are applied, where the parallel ion velocity is set according to the Chodura criterion [29, 30] and the condition on the electron velocity is used in linearised form. Via Ohm's law (equation (10)) we obtain a condition for the electrostatic potential, which is measured with respect to the sheath floating potential $\Lambda=$ $\frac{1}{2} \log \left(\frac{m_{i}}{2 \pi m_{e}}\right)$. Finally, outflow conditions for the density and vorticity are set.

$$
\begin{aligned}
\left.u_{\|}^{i}\right|_{X} & \gtrless \pm 1, \\
\left.j_{\|}\right|_{X} & = \pm n \phi, \\
\nabla_{\|} \phi \pm\left.\frac{n}{\sigma} \phi\right|_{X} & =\nabla_{\|} \log n \\
\left.\nabla_{\|}^{K} \log n\right|_{X} & =0, \\
\left.\nabla_{\|}^{K} \Omega\right|_{X} & =0,
\end{aligned}
$$

where the upper/lower sign applies if the magnetic field is directed towards/away from the target plate. $K$ is an integer which specifies the order of extrapolation of the outflow boundary condition, and due to technical reasons (see section 3 ) we set the outflow condition on $\log n$ instead of $n$. For the sake of robusteness the employed boundary conditions are kept relatively simple. A detailed discussion on boundary conditions for fluid SOL turbulence codes can be found in [31], and the implementation of the therein given boundary conditions, which also take into account an inclination of the magnetic field with respect to the material plates, is considered for future work.

\section{Numerical approach and implementation}

A general introduction into the Flux-Coordinate Independent (FCI) approach can be found in [1, 2]. In its basic form (see fig. 1) the simulation domain of e.g. a tokamak is spanned with a cylindric grid $\left(R_{i}, \varphi_{k}, Z_{j}\right)$. Within poloidal planes $\left(R_{i}, Z_{j}\right)$ the grid is Cartesian and bound by limiting flux surfaces and divertor or limiter plates (see fig. 1). Based on the assumption of a strong toroidal field $\left(B_{t o r} \gg B_{p o l}\right)$ perpendicular operators can be easily discretised with e.g. standard finite difference methods within the Cartesian poloidal planes. The discretisation of parallel operators follows the idea of a finite difference along magnetic field lines, i.e. from each grid points field lines are traced to adjacent poloidal planes and values at the obtained map points are computed via interpolation.

Several numerical practices were developed for the FCI in the past: Based on the method of support operators [32, 33] a self-adjoint discretisation for the parallel diffusion operator was derived which exhibits a very low level of numerical perpendicular diffusion $[2,17]$. For strongly distorted maps a combination of integration with interpolation was suggested [18]. Finally, the subtle but critical issue of treatment of boundaries within FCI was touched upon in $[18,20]$. All these findings with small modifications, which will be discussed in the subsequent sections, went into the development of GRILLIX. 


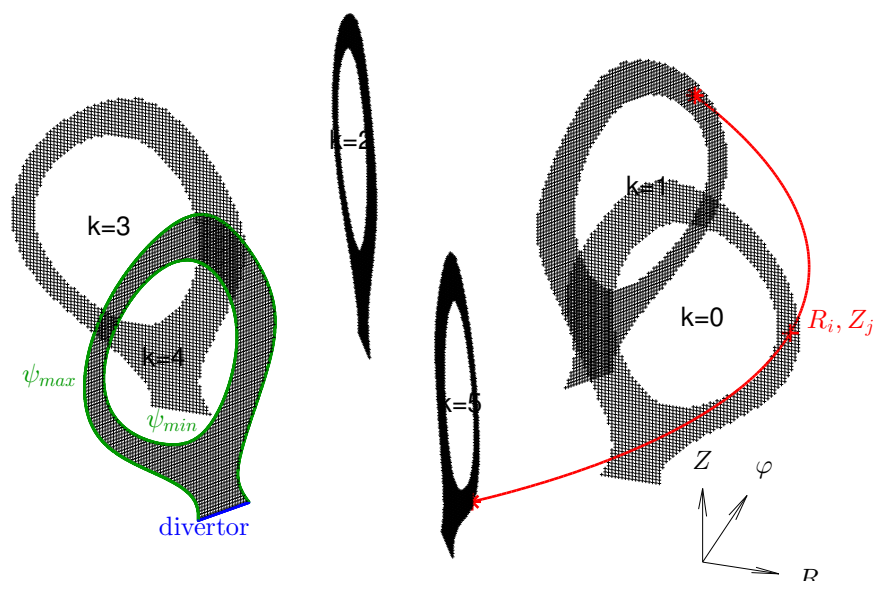

Figure 1: General overview of FCI concept in tokamak geometry. Grid, i.e. set of Cartesian poloidal planes, is limited by outer $\left(\psi_{\max }\right)$ and optionally inner $\left(\psi_{\min }\right)$ flux surface and divertor plates. Parallel operators are discretised via field line map, i.e. field line tracing and interpolation.

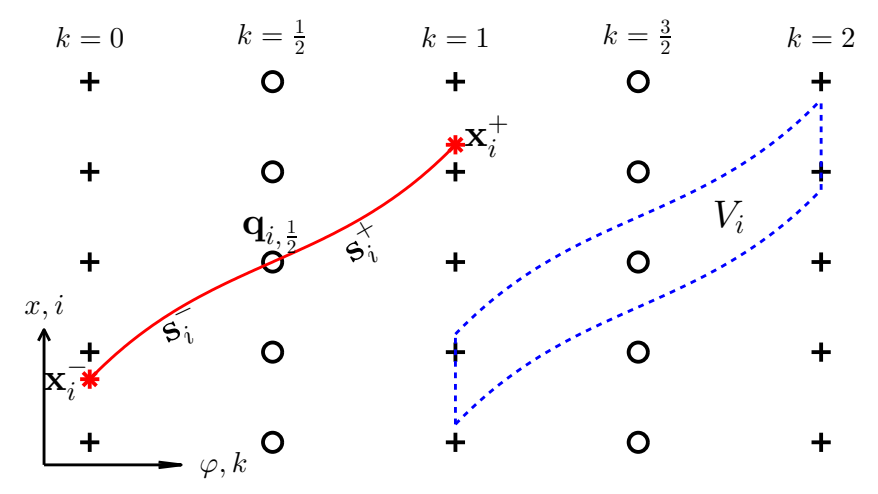

Figure 2: Scheme for toroidally staggered FCI approach. Grid is indicated with crosses and dual grid with circles.

\subsection{Toroidally staggered FCI}

A conservative formulation of the FCI was presented in [2], where the parallel gradient $\nabla_{\|}$and the parallel divergence $\nabla \cdot(\mathbf{b} \circ)$ fulfil the adjointness relation on the discrete level. We present a modification which is based on a toroidally staggered grid instead of a parallelly staggered grid. This resolves a strange peculiarity of [2] where two distinct discretisations $\left({ }^{\prime} \pm^{\prime}\right)$ for the same operator arose. In the following we will restrict the discussion without loss of generality to two dimensions $(x, \varphi)$, where $x$ can be interpreted as a poloidal angle and $\varphi$ as the toroidal angle. The magnetic field is assumed to be axisymmetric, i.e. independent of $\varphi$.

The scheme for the toroidally staggered FCI scheme is illustrated in fig. 2. In addition to the canonical grid $G$ denoted with integer toroidal grid indices $(k=0,1,2, \ldots)$ a dual grid $G^{*}$ with half-integral toroidal grid indices $\left(k=\frac{1}{2}, \frac{3}{2}, \frac{5}{2}, \ldots\right)$ is introduced. We define the discrete parallel gradient $\mathbf{Q}_{X}$ as an operator which maps from the grid to the dual grid:

$$
\mathbf{Q}_{X}: G \rightarrow G^{*},
$$

where the integer subscript $X$ will be clarified later. For some quantity $\mathbf{u}$ collocated on $G$ its discrete parallel gradient $\mathbf{q}$ collocated on $G^{*}$ is defined (in the case $X=0$ ) as:

$$
\mathbf{q}_{i, k+\frac{1}{2}}:=\left(\mathbf{Q}_{0} \mathbf{u}\right)_{i, k}:=\frac{u_{k+1}\left(x_{i}^{+}\right)-u_{k}\left(x_{i}^{-}\right)}{s_{i}^{+}+s_{i}^{-}},
$$

where $x_{i}^{ \pm}$are map points and $s_{i}^{ \pm}$lengths along field lines, which are due to the assumed axisymmetry independent of the grid index $k$. Values at map points $u_{k}\left(x_{i}^{ \pm}\right)$are obtained via interpolation within the poloidal plane $k$, where the coefficients of interpolation are contained in the matrix $\mathbf{Q}_{0}$. The definition of the discrete parallel gradient (28) can be generalised to a combination of integration and interpolation, where the parameter $X$ somewhat controls the amount of integration. This is especially important for strongly distorted maps, but we pass on a detailed description of this which follows in complete analogy to [18], where an exact definition for the parameter $X$ can be found.

The discrete version $\mathbf{Q}_{X}^{*}$ of the parallel divergence $\nabla \cdot(\mathbf{b} \circ)$ follows in analogy to $[2,17]$ according to the method of support operators $[32,33]$. Neglecting surface terms the following integral equality holds true:

$$
\int u \nabla \cdot\left(\mathbf{b} \nabla_{\|} v\right) d V=-\int \nabla_{\|} u \nabla_{\|} v d V,
$$

which we mimic on the discrete level as:

$$
\sum_{\alpha, \beta, \gamma} \mathbf{u}_{\alpha} \mathbf{Q}_{X, \alpha, \beta}^{*} \mathbf{Q}_{X, \beta, \gamma} \mathbf{v}_{\gamma} \mathbf{V}_{\alpha}=-\sum_{\mu, \nu, \tau} \mathbf{Q}_{X, \mu, \nu} \mathbf{u}_{\nu} \mathbf{Q}_{X, \mu, \tau} \mathbf{v}_{\tau} \mathbf{V}_{\mu}
$$

where Greek indices denote a summation over all grid points and $\mathbf{V}$ is a diagonal matrix containing flux box volumes $V_{i}$ as entries as illustrated in fig. 2 with dashed blue lines. After relabelling the indices the discrete parallel divergence follows as:

$$
\mathbf{Q}_{X}^{*}: G^{*} \rightarrow G, \quad \mathbf{Q}_{X}^{*}=-\mathbf{V}^{-1} \mathbf{Q}_{X}^{T} \mathbf{V} .
$$

The discrete operators $\mathbf{Q}_{X}$ and $\mathbf{Q}_{X}^{*}$ fulfil by construction the adjointness relation (29) on the discrete level.

Finally, we will need operators $\mathbf{M}, \mathbf{M}^{*}$ which map a quantity from the grid $G$ to the dual grid $G^{*}$ and vice versa. We employ a simple definition using a linear interpolation along magnetic field lines.

$$
\begin{aligned}
\mathbf{M}: G \rightarrow G^{*}, \quad(\mathbf{M u})_{i, k}:= & \frac{s_{i}^{-} u_{k+1}\left(x_{i}^{+}\right)+s_{i}^{+} u_{k}\left(x_{i}^{-}\right)}{s_{i}^{+}+s_{i}^{-}}, \\
\mathbf{M}^{*}: G^{*} \rightarrow G, \quad\left(\mathbf{M}^{*} \mathbf{u}^{*}\right)_{i, k}: & :=\frac{s_{i}^{-} u_{k+\frac{1}{2}}^{*}\left(x_{i}^{+}\right)+s_{i}^{+} u_{k-\frac{1}{2}}^{*}\left(x_{i}^{-}\right)}{s_{i}^{+}+s_{i}^{-}},
\end{aligned}
$$

where $\mathbf{u}^{*}$ is a quantity located on the dual grid.

\subsection{Discrete equations}

We will now write down the equations $(8,9,10,13)$ of the physical model in spatially discrete form, and the temporal discretisation will be discussed in the next section.

Based again on the assumption of a strong toroidal field any perpendicular operator can be approximated as operator acting purely within a poloidal plane, i.e. $\mathbf{u}_{E} \cdot \nabla f \approx$ 
$-B^{-1}\left(\partial_{R} \phi \partial_{Z} f-\partial_{Z} \phi \partial_{R} f\right)$ and $C(f) \approx-2 \partial_{Z} f$ where the Jacobi bracket $[\phi, f]:=\partial_{R} \phi \partial_{Z} f-\partial_{Z} \phi \partial_{R} f$ is discretised with the Arakawa scheme from [34] and for all other perpendicular/poloidal operators standard second order finite difference methods are employed.

In order to ensure its positivity the logarithm of the density $\theta_{n}:=\log n$ is developed in time. The structure of the equations suggests that the discrete density $\mathbf{n}$, its $\operatorname{logarithm} \boldsymbol{\theta}_{\boldsymbol{n}}$, potential $\phi$ and vorticity $\Omega$ are collocated on the canonical grid $G$, whereas the discrete parallel current $\mathbf{j}_{\|}^{*}$ and the discrete parallel ion velocity $\mathbf{u}_{\|}^{*}$ are collocated on the dual grid $G^{*}$. The spatially discrete equations are:

$$
\begin{aligned}
\frac{\partial}{\partial t} \boldsymbol{\theta}_{\boldsymbol{n}} & -\frac{\delta}{B}\left[\boldsymbol{\phi}, \boldsymbol{\theta}_{\boldsymbol{n}}\right]+\mathbf{M}^{*}\left(\mathbf{u}_{\|}^{*} \mathbf{Q}_{X} \boldsymbol{\theta}_{\boldsymbol{n}}\right)+\mathbf{Q}_{X}^{*} \mathbf{u}_{\|}^{*} \\
& =\mathbf{C}\left(\boldsymbol{\phi}-\boldsymbol{\theta}_{\boldsymbol{n}}\right)+\frac{1}{\mathbf{n}} \mathbf{Q}_{X}^{*} \mathbf{j}_{\|}^{*}+\frac{1}{\mathbf{n}} S_{n}+\frac{1}{\mathbf{n}}\left(\mathbf{D}_{\perp}^{n} \mathbf{n}+\mathbf{D}_{\|}^{n} \mathbf{n}\right), \\
\frac{\partial}{\partial t} \mathbf{u}_{\|}^{*} & -\frac{\delta}{B}\left[\mathbf{M} \boldsymbol{\phi}, \mathbf{u}_{\|}^{*}\right]+\mathbf{u}_{\|}^{*} \mathbf{Q}_{X} \mathbf{M}^{*} \mathbf{u}_{\|}^{*} \\
& =-\mathbf{Q}_{X} \boldsymbol{\theta}_{\boldsymbol{n}}-\frac{\mathbf{u}_{\|}^{*} S_{n}}{\mathbf{M n}}+\mathbf{D}_{\perp}^{u} \mathbf{u}_{\|}^{*}+\mathbf{D}_{\|}^{u} \mathbf{u}_{\|}^{*}, \\
\frac{\partial}{\partial t} \boldsymbol{\Omega} & -\frac{\delta}{B}[\boldsymbol{\phi}, \boldsymbol{\Omega}]=\mathbf{\Omega C} \boldsymbol{\phi}-\mathbf{C n}+\mathbf{Q}_{X}^{*} \mathbf{j}_{\|}^{*}+\mathbf{D}_{\perp}^{\Omega} \mathbf{\Omega}, \\
\mathbf{\Omega} & =\mathbf{P} \boldsymbol{\phi},
\end{aligned}
$$

with the matrix $\mathbf{P}$ the discrete analogue of $\nabla \cdot\left(B^{-2} \nabla_{\perp} \circ\right), \mathbf{D}_{\perp}^{f}$ of the perpendicular dissipation and $\mathbf{C}$ of the curvature operator . The discrete parallel diffusion operator is $\mathbf{D}_{\|}^{f}:=\mathbf{Q}_{X}^{*} \mu_{f} \mathbf{Q}_{X}$.

\subsection{Time-stepping}

The Karniadakis multi-step method from [35] is used for time advancement. We split the equation system $\partial_{t} \mathbf{F}=f(\mathbf{F}, t)$ into a part which is treated explicitly $f^{e}$ and implicitly $f^{i}$. The state at time step $t+1$ is computed according to:

$$
\begin{aligned}
\mathbf{F}^{t+1} & -\frac{6}{11} \Delta t f^{i}\left(\mathbf{F}^{t+1}, t+\Delta t\right)=\frac{18}{11} \mathbf{F}^{t}-\frac{9}{11} \mathbf{F}^{t-1}+\frac{2}{11} \mathbf{F}^{t-2} \\
& +\frac{6}{11} \Delta t\left[3 f^{e}\left(\mathbf{F}^{t}, t\right)-3 f^{e}\left(\mathbf{F}^{t-1}, t-\Delta t\right)+f^{e}\left(\mathbf{F}^{t-2}, t-2 \Delta t\right)\right]
\end{aligned}
$$

with $\Delta t$ the size of the time step. Terms related with the parallel current pose a strict requirement on the size of the time step [36] and are therefore treated implicitly on the left hand side contained in $f^{i}$. All other terms are treated in an explicit manner contained in $f^{e}$ on the right hand side. The resulting linear equation system which is solved in GRILLIX in each time step can symbolically be written as:

$$
\left(\begin{array}{cccc}
\mathbf{1} & 0 & -\frac{6}{11} \frac{1}{\mathbf{n}^{t+1}} \Delta t \mathbf{Q}_{X}^{*} & 0 \\
0 & \mathbf{1} & 0 & 0 \\
-\sigma \mathbf{Q}_{X} & 0 & \mathbf{1} & \sigma \mathbf{Q}_{X} \\
0 & 0 & -\frac{6}{11} \Delta t \mathbf{Q}_{X}^{*} & \mathbf{P}
\end{array}\right)\left(\begin{array}{l}
\boldsymbol{\theta}_{n}^{t+1} \\
\mathbf{u}_{\|}^{* t+1} \\
\mathbf{j}_{\|}^{* t+1} \\
\boldsymbol{\phi}^{t+1}
\end{array}\right)=\left(\begin{array}{c}
\mathbf{b}_{\theta_{n}} \\
\mathbf{b}_{u_{\|}} \\
0 \\
\mathbf{b}_{\Omega}
\end{array}\right),
$$

with $\mathbf{b}_{f}$ the corresponding right hand sides according to the time step scheme (39), which contains also all explicitly treated

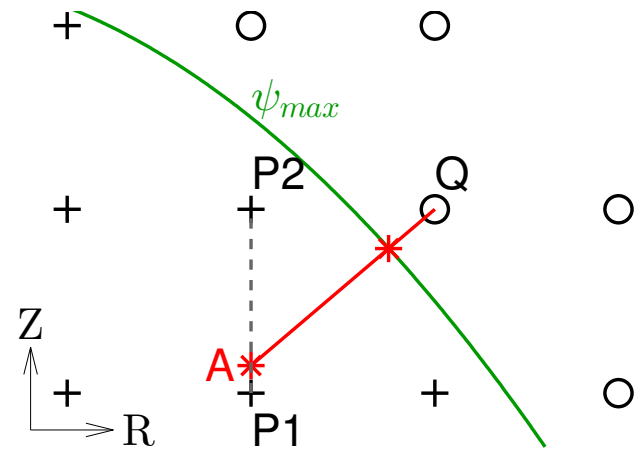

Figure 3: Scheme for treatment of radial boundary conditions. Crosses mark positions of grid and circles of ghost grid. For homogeneous Neumann boundary conditions the value of ghost point $\mathrm{Q}$ is set to the value at point $\mathrm{A}$, which is obtained from linear interpolation between points $\mathrm{P} 1$ and $\mathrm{P} 2$.

terms. A difficulty arises due to the factor $\frac{1}{\mathbf{n}^{t+1}}$ in the matrix which makes the problem non-linear. In order to obtain a linear problem we use here the extrapolated value obtained from the 3 previous time steps.

\subsection{Treatment of boundaries}

For the treatment of boundaries we proceed with the approach based on ghost points presented in [18].

Firstly we discuss the treatment of radial boundaries at $\psi_{\text {min }}$ and $\psi_{\max }$, which are cumbersome since the boundaries do not conform with the grid. For the elliptic problem $\nabla \cdot\left(B^{-2} \nabla_{\perp} \phi\right)=$ $\Omega$ with Dirichlet boundary conditions we use the ShortleyWeller scheme [37] and the case of homogeneous Neumann boundary conditions is treated according to fig. 3. We extend the grid within the poloidal plane by ghost points outside the limiting flux surface. For some ghost point $\mathrm{Q}$ the normal to the boundary contour is determined and its point of interesction A with the nearest horizontal respectively vertical (depending which one is closer) grid line. The value of ghost point $Q$ is then set to the value at the intersection point $\mathrm{A}$, which is obtained from linear interpolation between point P1 and P2. We note that this is a numerically poor zeroth order procedure and more sophisticated methods exist (e.g. [38]), which treat the boundary conditions more accurately. However, their implementation is cumbersome and intended for future work. Moreover, for parallel operators we also decrease the order of interpolation near the radial boundaries such that the interpolation stencil is always complete.

The treatment of parallel boundary conditions, i.e. at divertor/limiter plates, is also based on ghost points and we will restrict the discussion here again to two dimensions. The grid is supplemented by ghost points within the divertor/limiter region, and the extent of the parallel ghost grid must be sufficient such that the interpolation stencil of parallel operators for each inner grid point is complete. A specified boundary condition at the intersection of magnetic field lines with the target plate is set by assigning values to ghost points according to a Taylor expansion along magnetic field lines around the intersection point. In fig. 4 field line tracing from ghost point $\mathrm{A}$ is performed yielding the intersection point $\mathrm{X}$, its map point $\mathrm{B}$ and its next 


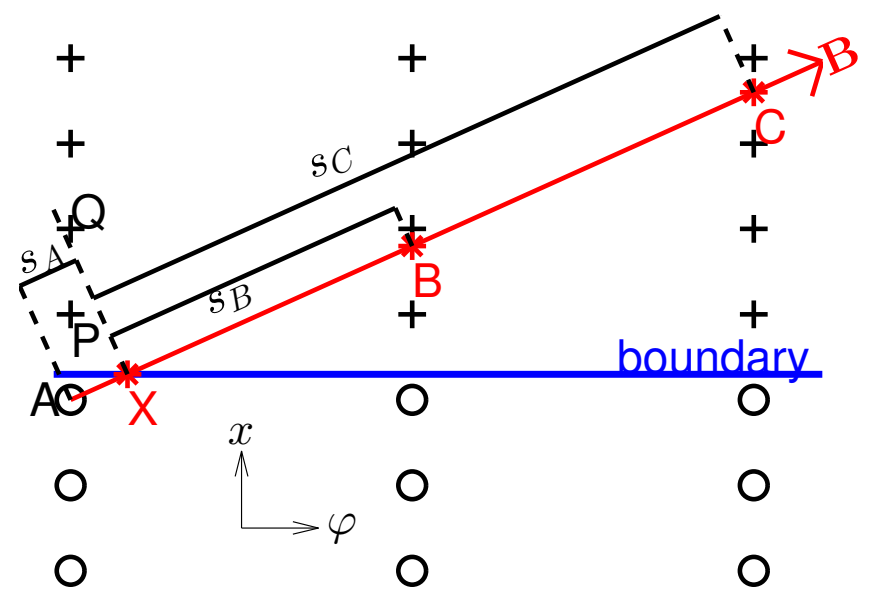

Figure 4: Scheme for treatment of parallel boundary conditions. Crosses mark positions of grid and circles of ghost grid.

but one map point $\mathrm{C}$. In addition the distances $s_{A}, s_{B}$ and $s_{C}$ (signed w.r.t. direction of magnetic field) of each point to the intersection point are computed. As an example for a Neumann boundary condition $\left.\nabla_{\|} u\right|_{X}=\alpha$ the value of ghost point A would be set according to a second order Taylor expansion as:

$$
u_{A}=\frac{s_{C}^{2}-s_{A}^{2}}{s_{B}^{2}-s_{C}^{2}} u_{B}+\frac{s_{A}^{2}-s_{B}^{2}}{s_{B}^{2}-s_{C}^{2}} u_{C}+\frac{\left(s_{A}-s_{B}\right)\left(s_{A}-s_{C}\right)}{s_{B}+s_{C}} \alpha,
$$

where the values $u_{B}$ and $u_{C}$ are obtained via interpolation. The treatment of the full parallel boundary conditions (22-26) follows in principle the same approach but is more elaborate and therefore discussed in Appendix B, and special care must also be taken for quantities defined on the dual grid $G^{*}$ which is also discussed in Appendix B.

A final subtlety regards the fact that ghost point $\mathrm{A}$ in fig. 4 is set completely independent of its poloidal neighbour $\mathrm{P}$ within the grid, and therefore a corrugation among point $\mathrm{A}$ and $\mathrm{P}$ might be present. This could lead to an overshot at the interpolation around the boundary (Runge's phenomenon), which caused sometimes even numerical instabilities in turbulence simulations. Therefore, we implemented the possibility to smooth the first inner grid point by applying after each time step to point $\mathrm{P}$ :

$$
u_{P}=(1-2 \epsilon) u_{P}+\epsilon\left(u_{A}+u_{Q}\right),
$$

where we typically choose $\epsilon \lesssim 0.1$.

\subsection{Comments on implementation}

GRILLIX evolves the discrete equation system (34-38) in time according to the scheme described in section 3.3. There are no obvious reasons why the developed methods should not easily be applicable to 3D configurations [20], e.g. stellarators, but GRILLIX is written as an axisymmetric code in order to facilitate implementation.

From the geometrical side GRILLIX takes as input the axisymmetric field $\mathbf{B}(R, Z)$, and the the position of the boundaries is specified via a level set function whose contours usually overlap with the poloidal flux function $\psi(R, Z)$. Via the

\begin{tabular}{l|ccccccc} 
Number of threads & 1 & 2 & 4 & 8 & 16 & 34 & 68 \\
Strong scaling [\%] & 100 & 94 & 87 & 80 & 65 & 45 & 27
\end{tabular}

Table 1: Strong scaling of OpenMP parallelisation for test problem with $\approx$ $2.5 \cdot 10^{5}$ grid points. Test was executed on MARCONI A2 partition with Knights Landing architecture (w/o hyper-threading).

level set function a Cartesian grid $\left(R_{i}, Z_{j}\right)$ is masked and only points within the mask are kept in memory. The grid is then supplemented by poloidal and parallel ghost points.

In a preprocessing step the map matrices $\mathbf{Q}_{X}, \mathbf{M}$ and $\mathbf{M}^{*}$ are built. Since the map is assumed to be time-independent this possibly costly task has to be performed only once at the beginning of a simulation and the result can even be written to hard disk for reuse. The field line tracing is performed via the DOP853 ODE Solver [39, 40], and the coefficients of interpolation are computed according to a polynomial interpolation [41] of arbitrary odd order (usually 3rd order) where the map point is always centred around the interpolation stencil. The final matrices are generally sparse and due to axisymmetry their blocks are identical for each plane $k$. Some further meta data like the flux box volumes and information related with setting of boundary conditions are also computed during the preprocessing phase.

The main task within the time loop is to solve the linear equation system (40). The computation of the explicitly treated parts, i.e. the right hand side of equation (40), is straight forward, and the equation system is then solved via a GMRES algorithm based on a reverse communication interface [42]. In order to speed up computation we use as preconditioner:

$$
\mathbf{R}:=\left(\begin{array}{cccc}
\mathbf{1} & 0 & 0 & 0 \\
0 & \mathbf{1} & 0 & 0 \\
-\sigma \mathbf{Q}_{X} & 0 & \mathbf{1} & \sigma \mathbf{Q}_{X} \\
0 & 0 & 0 & \mathbf{P}
\end{array}\right)
$$

i.e. terms proportional to $\Delta t$ are simply dropped. The preconditioning step becomes trivial if the elliptic problem $\mathbf{P x}=\mathbf{b}$ with $\mathbf{x}$ unknown is solved, for which we implemented a geometric multi-grid solver [37]. With this solver GRILLIX is already equipped for relaxation of the Boussinesq approximation in a computationally efficient way.

GRILLIX is MPI parallelised over the toroidal direction, i.e. each MPI process works on a single plane $k$. Due to axisymmetry each process executes the same amount of work and therefore load balancing is by construction excellent. Besides few global communications mainly for diagnostics purposes, the main communication takes place between adjacent planes for evaluation of parallel operators. Within each plane GRILLIX is OpenMP parallelised, respectively multi-threaded libraries are employed. Due to the field line map the connection between points (stencil) is in general rather complex and depends on the specific resolution and geometry employed. This is disadvantageous concerning memory access times on NUMA systems, and an example for the strong scaling of the OpenMP parallelisation is given in table 1 , which becomes at a large number of threads somewhat poor. 


\section{Verification and validation}

\subsection{Parallel transport in SOL}

Verification tests of GRILLIX concerning parallel diffusion as an isolated sub-problem can be found in [2, 17, 18]. As another important isolated sub-problem we give here an example for the parallel advective transport model in the SOL, in order to verify implementation of the Chodura boundary conditions in a sub- and super-sonic case. We consider the following subproblem:

$$
\begin{aligned}
& \frac{\partial}{\partial t} \theta_{n}+u_{\|}^{i} \nabla_{\|} \theta_{n}+\nabla \cdot\left(\mathbf{b} u_{\|}^{i}\right)=\frac{1}{n}\left(\mathcal{D}_{\|}(n)+\mathcal{D}_{\perp}(n)\right), \\
& \frac{\partial}{\partial t} u_{\|}^{i}+u_{\|}^{i} \nabla_{\|} u_{\|}^{i}=-\nabla_{\|} \theta_{n}+\mathcal{D}_{\|}\left(u_{\|}^{i}\right)+\mathcal{D}_{\perp}\left(u_{\|}^{i}\right),
\end{aligned}
$$

where the dissipation here is applied for numerical reasons, and Chodura boundary conditions (equations (22) and (25) with $K=2$ ) are applied at the target plates.

As an example we use a circular equilibrium with limiter and constant safety factor $q=3$. The initial state is $u_{\|}^{i}(t=0)=0$ and for the density a constant background $n_{0}$ plus a field aligned Gaussian structure of amplitude $n_{\text {blob }}=1$ of isotropic perpendicular width $w_{\perp}=2.5 \cdot 10^{-2}$. We choose as toroidal resolution $\Delta \varphi=\frac{2 \pi}{16}$ and a poloidal resolution of $h=3 \cdot 10^{-3}$ (Perpendicular scales are measured here in $R_{0}$, i.e. $\delta=1$ ). In order to suppress zig-zag structures we employ a parallel numerical diffusion of $\mu_{n}=\mu_{u}=0.04$ in combination with a small perpendicular hyper-diffusion $\left(N=2, v_{n}=v_{u}=0.01\right)$. In fig. 5 the result of a simulation with $n_{0}=1$ is shown. The blob initially splits into two counter-propagating parts and outflow at the target plates is mostly sonic. Also the parallel ghost points are explicitly included in the figure in order to show that quantities are obviously smooth across the boundary (We used for this test $\epsilon=0$ ). In fig. 6 the result of a simulation with low background of $n_{0}=1 \cdot 10^{-2}$ is shown, where the blob propagates at supersonic speed. As it approaches the target plates the boundary condition for the parallel velocity changes from $\left.u_{\|}^{i}\right|_{X}= \pm 1$ to $\left.\nabla_{\|}^{2} u_{\|}^{i}\right|_{X}=0$, i.e. values on ghost points are then set via linear extrapolation along magnetic field lines (see Appendix B).

\subsection{Method of manufactured solutions}

We verify the implementation of the physical model in GRILLIX with the Method of Manufactured Solutions (MMS) [25], which has become a standard procedure in the community $[10,12,43,44]$. MMS can be performed at various levels of thoroughness, but we will restrict our test case here only to circular geometry on closed magnetic field lines with homogenous radial Dirichlet boundary conditions. A more extensive analysis is postponed to a later point in time, when a more complex physical model will be available in GRILLIX (see section 6).

We define the poloidal angle $\theta(R, Z):=\arctan \left(\frac{Z / \delta}{R / \delta-1}\right)$ and a flux surface label $\rho(R, Z):=\sqrt{(R / \delta-1)^{2}+(Z / \delta)^{2}}$ with the domain radially bounded by $\rho \in\left[\rho_{\min } \ldots \rho_{\max }\right]$. The magnetic field is prescribed in terms of a safety factor profile of the form $q(\rho)=q_{0}+s\left(\rho-\rho_{0}\right)$ with $q_{0}$ a reference value at the central a)
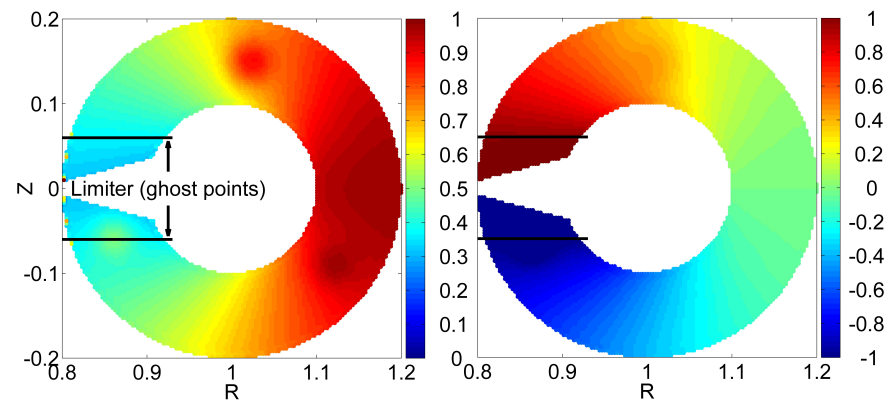

b)

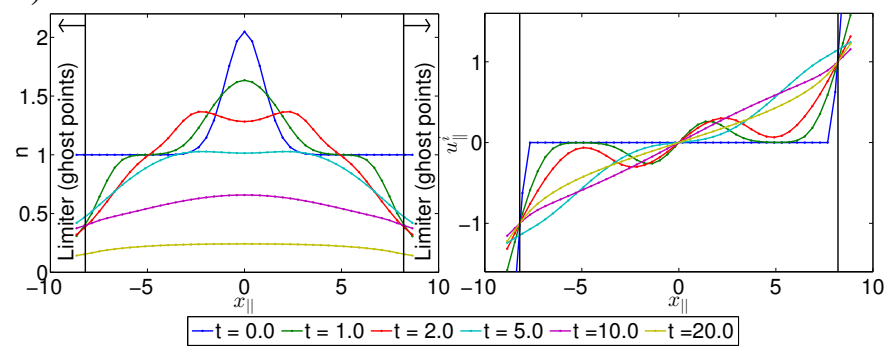

Figure 5: a) Poloidal cross section of density (left) and parallel velocity for blob with $n_{0}=1$ at $t=5.5, \varphi=2 \pi \frac{11}{16}$. Parallel ghost points are shown explicitly in between limiter plates (black lines). b) Density (left) and parallel velocity (right) along magnetic field line passing through center of blob at different times.

a)

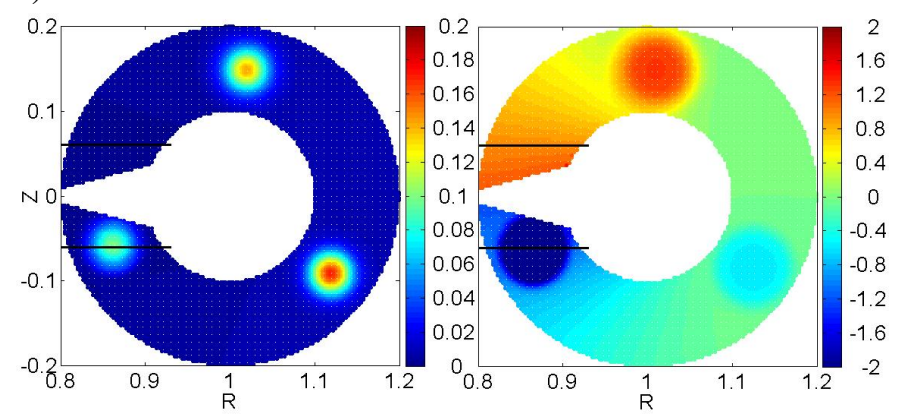

b)

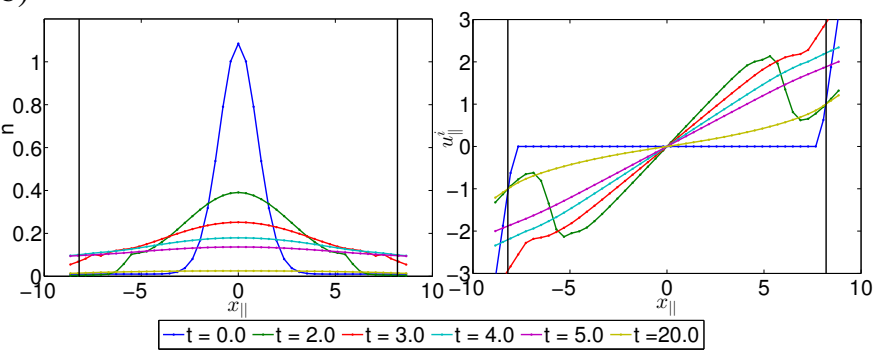

Figure 6: Same as fig. 5 for $n_{0}=1 \cdot 10^{-2}$. Snapshot is at $t=4.0$. 


\begin{tabular}{l|cccc} 
& $k_{\rho}$ & $k_{\theta}$ & $k_{\varphi}$ & $\omega$ \\
\hline$\theta_{n}$ & 1 & 1 & 1 & 100 \\
$\phi$ & 2 & 2 & 1 & 80 \\
$u_{\|}^{i}$ & 3 & 1 & 1 & 65
\end{tabular}

Table 2: Parameters used for MMS solution.

flux surface $\rho_{0}:=\left(\rho_{\min }+\rho_{\max }\right) / 2$ and $s$ the magnetic shear. As MMS solutions we choose functions of the following type:

$u_{M M S}(\rho, \theta, \varphi, t)=\sin \left(2 \pi k_{\rho} \frac{\rho-\rho_{\min }}{\rho_{\max }-\rho_{\min }}\right) \sin \left(k_{\theta} \theta\right) \cos \left(k_{\varphi} \varphi\right) \sin (\omega t)$,

which are rather general, as the discretisation of the FCI approach does not rely on flux/polar coordinates $\rho, \theta$ within poloidal planes but on Cartesian coordinates $R, Z$. The temporal discretisation error in GRILLIX is of third order in time according to the Karniadakis scheme. The spatial discretisation error is of second order except for grid points in direct vicinity of radial boundaries, where the Shortley-Weller scheme is used for the discretisation of the elliptic operator and the interpolation order might be reduced.

We benchmark the complete physical model $(8,9,10,13)$ without source $\left(S_{n}=0\right)$ and regular perpendicular diffusion $(N=0)$. A circular geometry with magnetic shear $\left(q_{0}=3\right.$, $s=10)$ is used where the domain ranges radially from $\rho_{\min }=$ 0.1 to $\rho_{\max }=0.18$. The simulation parameters are in the range of turbulence simulations presented later on, i.e. $\delta=680$, $\sigma=1, v_{n, u, \Omega}=13, \mu_{n, u}=1 \cdot 10^{-2}$, and the parameters of the MMS solutions are summarized in table 2 . We cover a resolution of $\Delta \varphi=\frac{2 \pi}{8} / 2^{i}$ with a poloidal resolution of correspondingly $h=2.04 / 2^{i}$ and time steps $\Delta t=1 \cdot 10^{-4} / 2^{i}$, where $i=0 \ldots 4$. The numerical error is measured in the $L 2$ norm $\Delta_{2}:=\left|u_{\text {num }}-u_{M M S}\right|_{2} /\left|u_{M M S}\right|_{2}$ at time $t=0.2$.

The result of the MMS procedure in fig. 7 shows a convergence at second order for all quantities. We do not observe any degradation of the convergence order due to the poorer numerical treatment at the radial boundaries, and the reason for this might be the use of homogeneous Dirichlet boundary conditions for this test case. The overall result provides strong confidence in the correct implementation of GRILLIX.

\subsection{TORPEX blob}

A simple validation exercise against the TORPEX experiment [45] and simultaneously a benchmark against several edge/SOL codes is based on the propagation of seeded blobs. The full details about the experimental setup and simulations carried out with STORM(BOUT++), GBS, TOKAM3X and HESEL can be found in [4]. The TORPEX device is modelled with GRILLIX in slab geometry $(R, \varphi, Z)$, where the magnetic field is aligned with the axial coordinate $\varphi$, i.e. $\mathbf{B}=\mathbf{e}_{\varphi}$. Sheath boundary conditions are applied at $\varphi=0$ and $\varphi=2 \pi$ and periodic boundary conditions in $y$-direction. In $x$-direction boundary values are set to the background values, which is obtained according to the procedure described in [46]. A snapshot of the

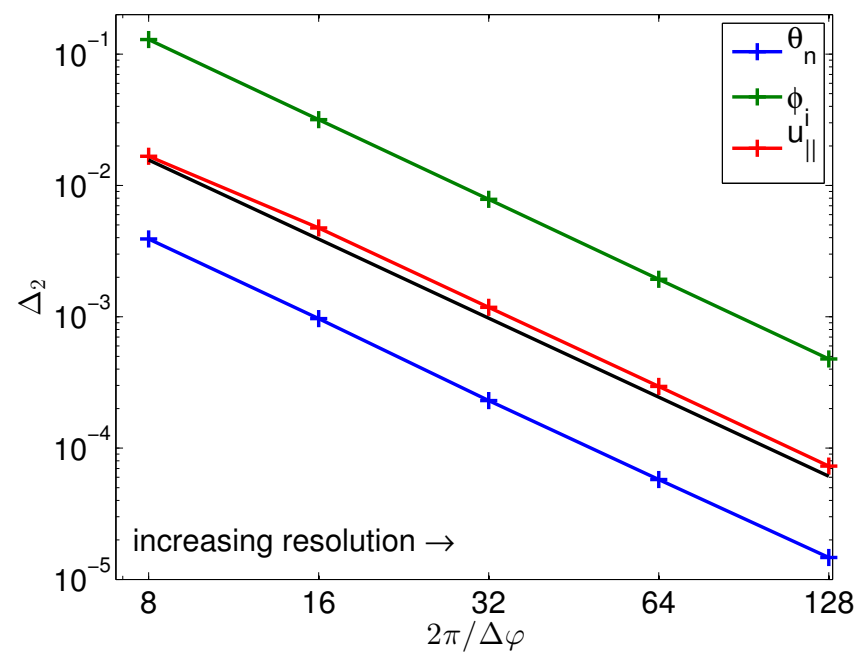

Figure 7: Numerical error of MMS procedure in circular geometry with magnetc shear. Black solid line shows second order as reference.
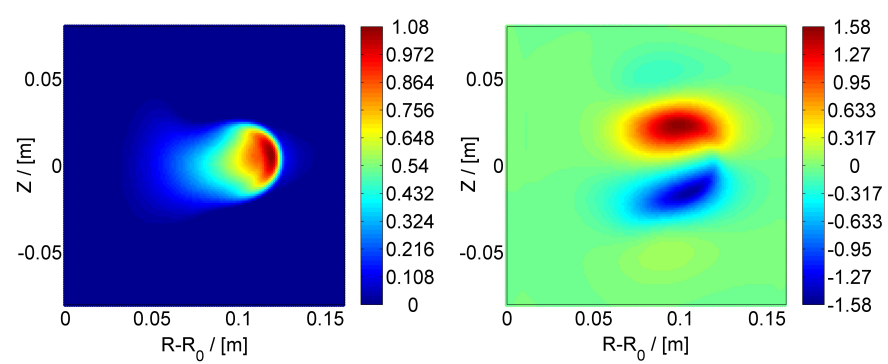

Figure 8: Snapshot of blob at $\varphi=0$ and $t=48 \mu \mathrm{s}$ for case 1 of [4]. Left: blob density $n-n_{b c k}$ in $10^{15} \mathrm{~m}^{-3}$, right: electrostatic potential $\phi$ in V. 
blob density and electrostatic potential for case 1 is shown in fig. 8 .

For a quantitative comparison we show in fig. 9 the radial and vertical velocities of the blob centroid. The radial velocities obtained with GRILLIX agree by trend with experimental data, whereas the vertical velocities are systematically underestimated. This deviation can be explained by an insufficiency of the isothermal physical model, especially missing of thermal effects [4] in GRILLIX and STORM. We compare to the STORM module (BOUT++3D) as it employs the same model as GRILLIX except that electron inertia is taken into account and the Boussinesq approximation is handled differently. The agreement between GRILLIX and STORM is good and the deviations are due to the mentioned differences in the physical model. In order to show this we have also implemented the Boussinesq approximation of STORM in GRILLIX, i.e. $\nabla \cdot\left(n \frac{d_{i}}{d t} \nabla_{\perp} \phi\right) \rightarrow n \frac{d_{i}}{d t} \Omega$ instead of (12). The agreement between the modified version GRILLIX(mod Bsq) and STORM is excellent, and small deviations in the vertical propagation velocities, especially for case 3 , could possibly be explained by effects due to electron inertia.

\section{Simulations}

The system is driven in GRILLIX by a particle source of the form

$$
S_{n}=c_{s r c} e^{-\left(\psi-\psi_{s r c}\right)^{2} / w_{s r c}^{2}}\left(n_{\text {target }}-\langle n\rangle_{\psi}\right),
$$

i.e. the source is located at the flux surface $\psi_{s r c}$ typically close to the inner limiting boundary with a Gaussian width of $w_{s r c}$ (typically a few $\rho_{s}$ ) and drives the zonal averaged density towards the target value $n_{\text {target }}$. We refer to Appendix $\mathrm{C}$ for the computation of flux surface averages within the FCI approach. In buffer zones close to the radial boundaries we additionally smooth all quantities by applying a strong perpendicular diffusion according to:

$$
\frac{\partial}{\partial t} u=\cdots+\nabla \cdot\left(v_{b f}(\psi) \nabla_{\perp} u\right)
$$

where we realize via step functions that only in the buffer zones $v_{b f}(\psi) \neq 0$.

\subsection{Closed magnetic flux surfaces}

For an example in tokamak geometry we prescribe the normalized magnetic field as:

$$
\mathbf{B}=\nabla \varphi+\nabla \varphi \times \nabla \psi(R, Z)
$$

a)

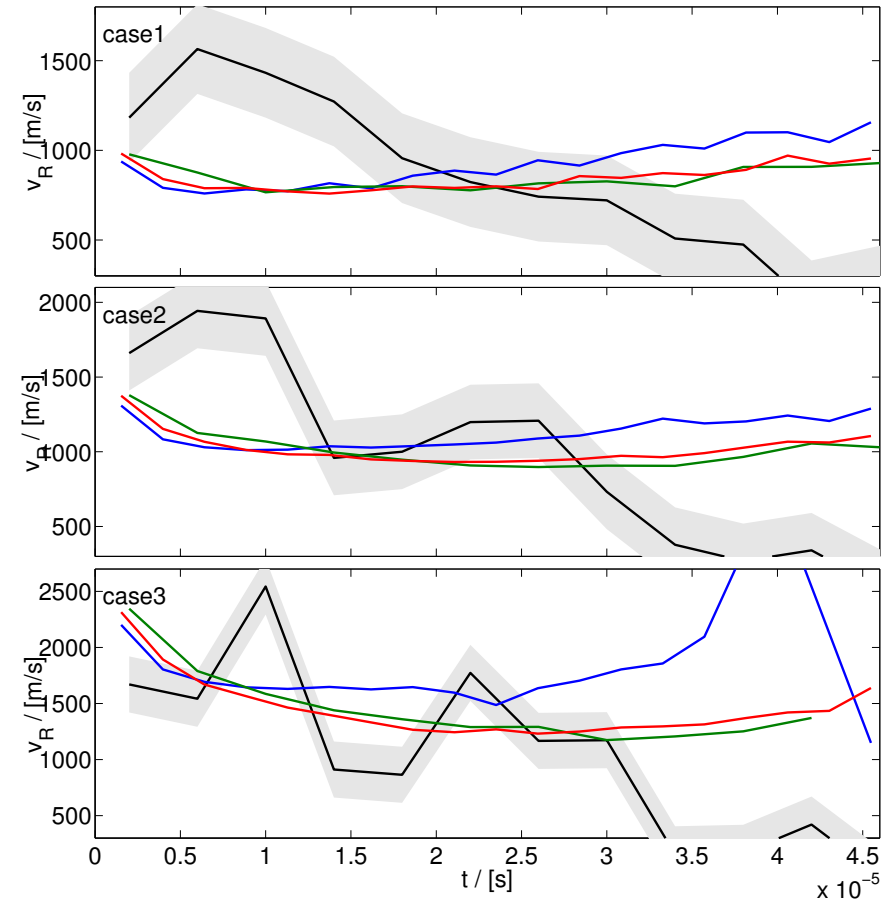

b)

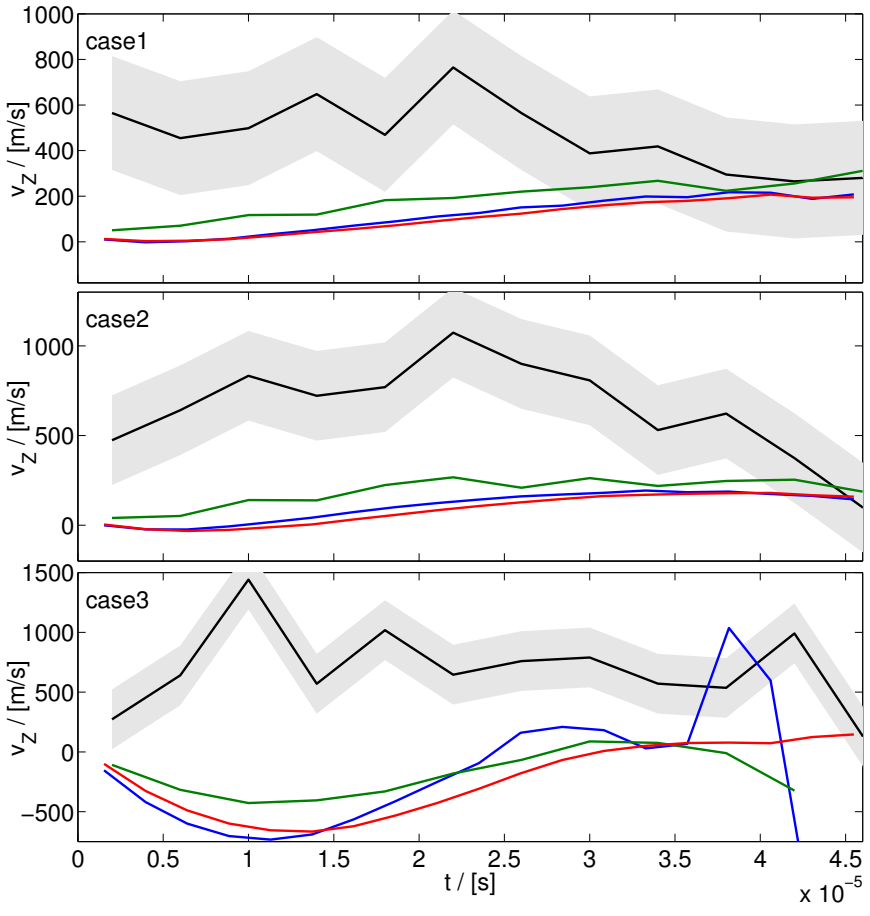

—Experiment -GRILLIX -STORM - GRILLIX (mod Bsq)

Figure 9: Radial (a) and vertical (b) velocities of TORPEX multi-code validation obtained with GRILLIX, STORM, experiment with uncertainty (grey shaded area) and the modified version GRILLIX(mod Bsq) which employs the same Boussinesq approximation as STORM. Experimental data and data of STORM was picked from [4], where also further details on the setup of case 1 (top), case 2 (center) and case 3 (bottom) can be found. 


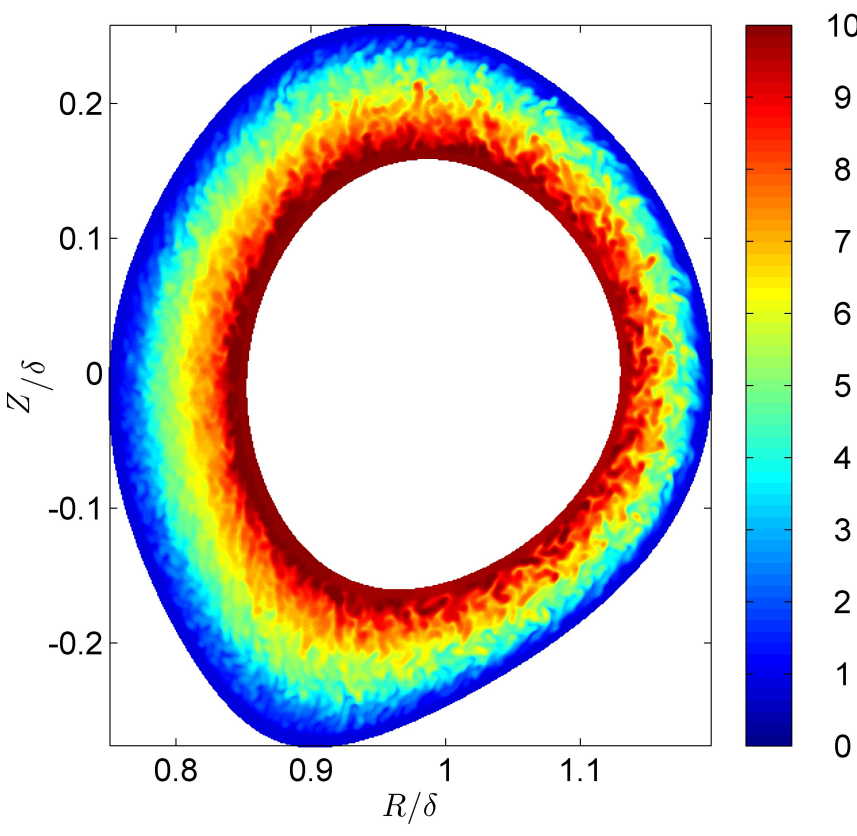

Figure 10: Snapshot of density $n$ at poloidal cross section $\varphi=0$ and time $t=53$. Resolution within poloidal plane was $h=1$, i.e. $\approx 65$ points radially at outboard mid-plane, and 16 poloidal planes were used. Time step was $d t=1 \cdot 10^{-4}$.

where we use as poloidal flux function an analytic solution to the Grad-Shafranov equation from the class of solutions of [47]:

$$
\begin{aligned}
\psi(\hat{R}, \hat{Z})= & 0.0159-0.0363 \hat{R}^{2}-0.00262 \hat{R} J_{1}(5.836 \hat{R}) \\
& -0.0117 \hat{R}(1.769 \hat{Z}-0.231) J_{1}(5.836 \hat{R}) \\
& -0.0665 \hat{R} Y_{1}(5.836 \hat{R}) \\
& -0.0461 \hat{R} J_{1}(4.669 \hat{R}) \cos (3.502 \hat{Z}-0.457) \\
& +0.0360 \hat{R} J_{1}(3.502 \hat{R}) \cos (4.669 \hat{Z}-0.610) \\
& +0.0218 \hat{R} J_{1}(0.584 \hat{R}) \cos (5.807 \hat{Z}-0.758) \\
& -0.0383 \hat{R} J_{1}(6.825 \hat{R}) \cosh (3.537 \hat{Z}-0.462) \\
& +0.0238 \hat{R} J_{1}(4.669 \hat{R}) \sin (3.502 \hat{Z}-0.457) \\
& -0.00926 \sin (5.836 \hat{Z}-0.762),
\end{aligned}
$$

with $J_{1}$ and $Y_{1}$ Bessel functions of the first respectively second kind, and $\hat{R}=R / \delta$ and $\hat{Z}=Z / \delta$ the major radius and the vertical coordinate measured in units of $R_{0}$. Additionally, we define the normalized flux label $\rho=\sqrt{\left(\psi-\psi_{0}\right) /\left(\psi_{x}-\psi_{0}\right)}$ with $\psi_{0}, \psi_{x}$ the poloidal flux at the magnetic axis respectively separatrix.

Firstly, we present a simulation on closed magnetic flux surfaces covering $\rho \in[0.6,0.9]$ with a safety factor in the range of $q=1.76 \ldots 2.47$. We choose as parameters $\delta=1000$ and $\sigma=50$, which could reflect a deuterium plasma with roughly $n_{0}=2 \cdot 10^{18} \mathrm{~m}^{-3}, T_{e}=10 \mathrm{eV}, R_{0}=1 \mathrm{~m}, B_{0}=0.5 \mathrm{~T}$, $\tau_{e i}=4.4 \cdot 10^{-7} \mathrm{~s}$. A perpendicular hyper-diffusion $(N=2)$ with $v_{n}=v_{u}=v_{\Omega}=10$ is applied and parallel diffusion coefficients are set to $\mu_{n}=\mu_{u}=0.15$. The simulation is initialised with the background density plus small random fluctuations, and all other quantities are set to zero. A snapshot of the density in the

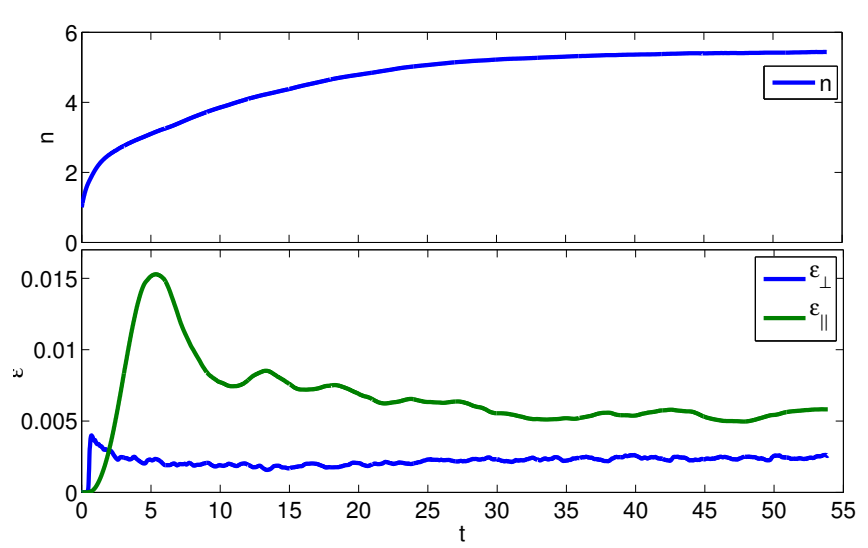

Figure 11: Volume averaged density (top) and perpendicular/parallel kinetic energy (bottom).

saturated state is shown in fig. 10 .

The volume averaged density, perpendicular and parallel kinetic energy are shown in fig. 11. Initially the density ramps quickly until at $t \approx 2$ the target density in the source region has already been reached. On a slower time scale density is then transported radially outwards via turbulent advection to the outer limiting flux surface, which constitutes a sink, until there is a balance between the driving source and the sink from around $t \approx 35$. In fig. 12 time derivatives of the volume averaged density and kinetic energies during the saturated phase are shown. It is obvious that the time derivative of the volume averaged density is a balance between the driving source and the sink at the outer limiting flux surface, showing that there are indeed no or at least only very small spurious numerical sources/sinks of particles in GRILLIX. The same is true for the perpendicular/parallel kinetic energies whose time derivatives are balanced by terms according to equations $(15,16)$. At this analysis we did not take into account the source term due to the polarisation drift in equation (16), showing that this term seems to be indeed very small.

As a basic diagnostics of the saturated state we show the average density profile and its radial gradient in fig. 13. The density decays from the target density in the source region to the background value at the outer limiting flux surface. In the average parallel current Pfirsch-Schlüter currents become obvious (see fig. 14), which are under the isothermal approximation proportional to the radial gradient of the density profile $j_{P S} \propto \partial_{\rho}\langle n\rangle_{\psi}$ [48]. The results of the simulation are consistent with this as regions of strong Pfirsch-Schlüter currents coincide with regions of strong density gradients at $\rho \approx 0.65,0.75,0.85$. We note that the magnetic field in GRILLIX is prescribed and fixed in time, but the average current and pressure profile evolve according to force balance $\langle\mathbf{j}\rangle_{\varphi, t} \times \mathbf{B}=\langle\nabla p\rangle_{\varphi, t}$. The root mean square density fluctuation exhibits the characteristic ballooning pattern with a high fluctuation level at the low field side, and a correlation between fluctuation level and density gradient is also observed.

The treatment of the magnetic axis is trivial within the FCI approach, and to show an example we consider a domain with 

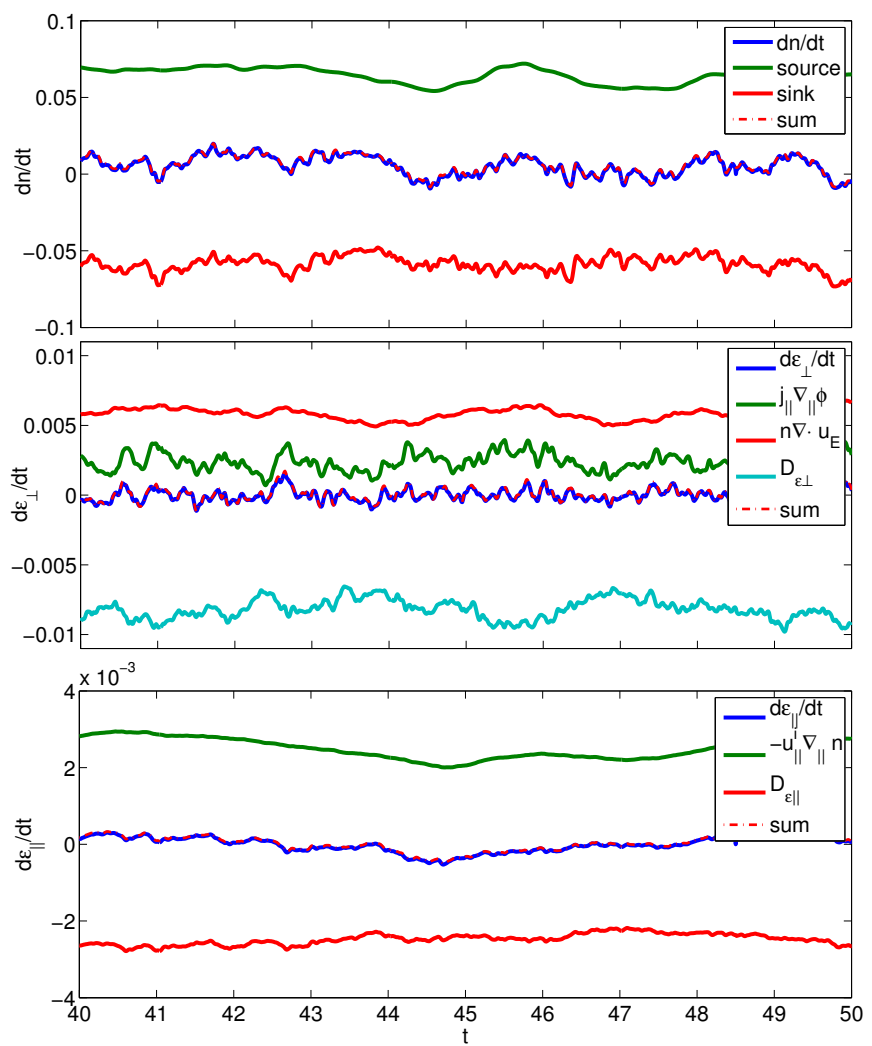

Figure 12: Time derivatives of volume averaged density (top), perpendicular kinetic energy (center) and parallel kinetic energy (bottom) during saturated phase. Solid blue lines represent directly computed time derivatives, i.e. obtained via finite difference between two adjacent time points. Other solid coloured lines are balancing terms of the underlying conservation theorem (see equations $(8,15,16))$ and dashed red line is sum of balancing terms.

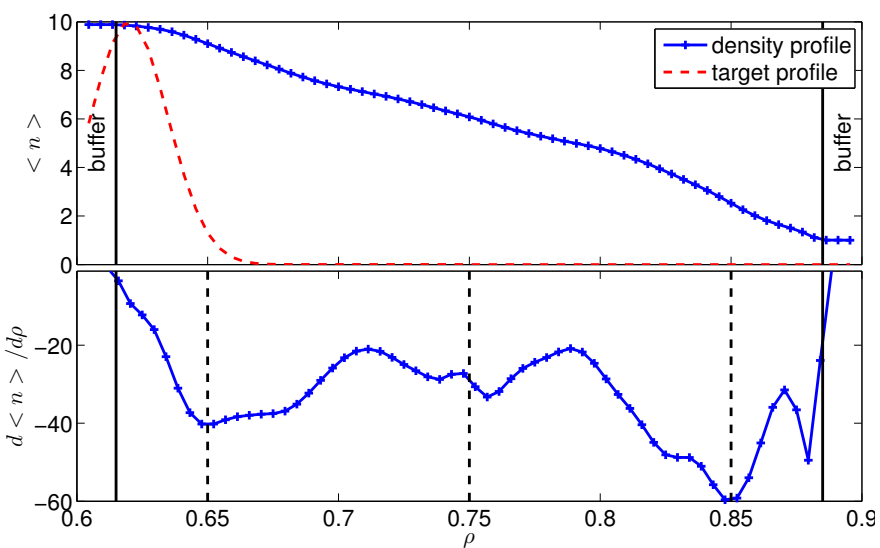

Figure 13: Density profile $\langle n\rangle_{\psi, t, \varphi}$ (top) averaged over $t=35 \ldots 53$. Solid black lines indicate buffer zones and red dashed line is profile of source $S_{n}$. Bottom: Radial gradient of density profile. Minima at around $\rho=0.65,0.75,0.85$ are indicated with dashed black lines.
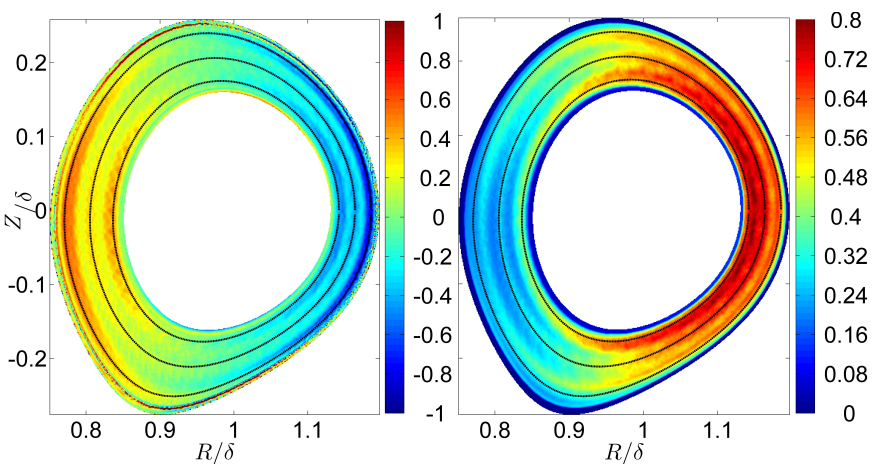

Figure 14: Average parallel current (left) and root men square of density fluctuations (right). Flux surfaces with strong density gradients (see fig. 13) are indicated with dashed black lines.
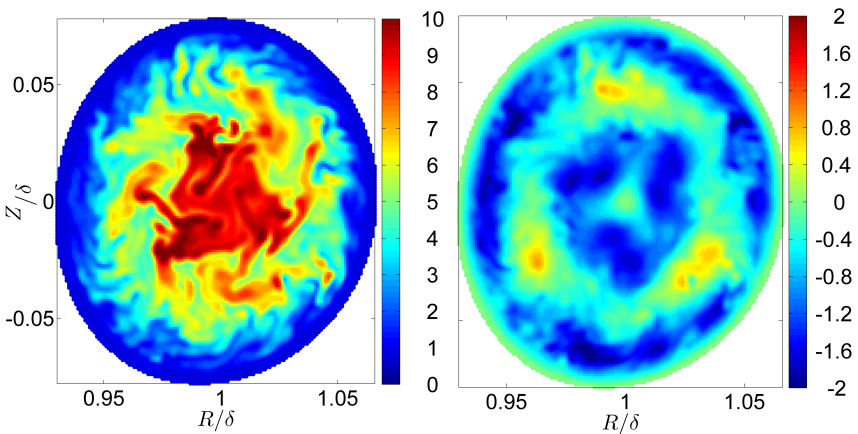

Figure 15: Snapshot of density (left) and electrostatic potential (right) for simulation including magnetic axis.

$\rho \in[0,0.3]$, where the source is located slightly off-axis at $\rho=$ 0.05 . A buffer zone is only present near the outer limiting flux surface and other parameters were taken over from the previous example. A snapshot of this simulation in the saturated phase is shown in fig. 15, where stable vortices in the potential have developed around the resonant surface with $q=3 / 2$.

\subsection{Limiter}

In a geometry with circular flux surfaces the magnetic field is prescribed via a safety factor profile $q(\rho)=q_{0}+s\left(\rho-\rho_{0}\right)$ with $\rho:=\sqrt{(R / \delta-1)^{2}+(Z / \delta)^{2}}$. We consider a pure SOL region with a toroidal limiter intersecting radially the full domain. A simultaneous treatment of open and closed field lines in limiter geometry is not yet available in GRILLIX, since an additional type of boundary would be present where field lines run tangentially to the limiter front plate.

For an example we use the same parameters as in the previous section 5.1 except that we reduced the parallel conductivity to $\sigma=10$ in order to speed up the computation. The simulation domain spans $\rho \in[0.018,0.03]$ with $q_{0}=2.5$ and $s=30$, i.e. the safety factor is in the range of $q=2.32 \ldots 2.68$. The direction of toroidal field is counter-clockwise and the direction of plasma current clockwise if viewed from above. A snapshot of the density in the saturated phase is shown in fig. 16 for a case with a toroidal limiter at the bottom and at the high field side. Blobs are observed and density is rather depleted 

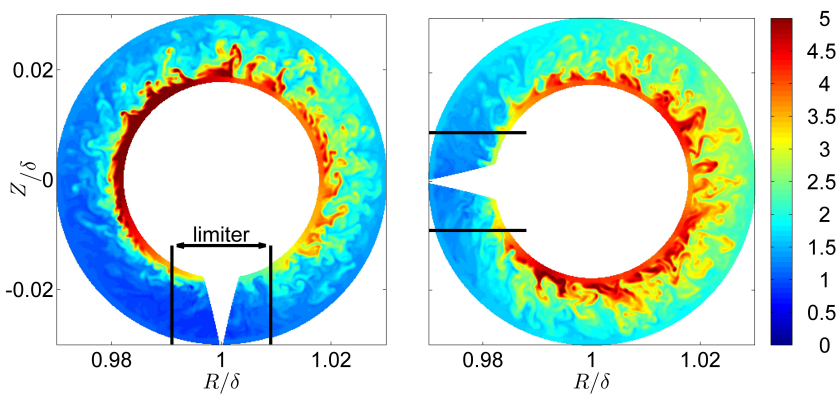

Figure 16: Snapshot of density at $\varphi=0$ in saturated phase of simulation with toroidal limiter at bottom (left) and at high field side (right). Ghost points between limiter plates are included.

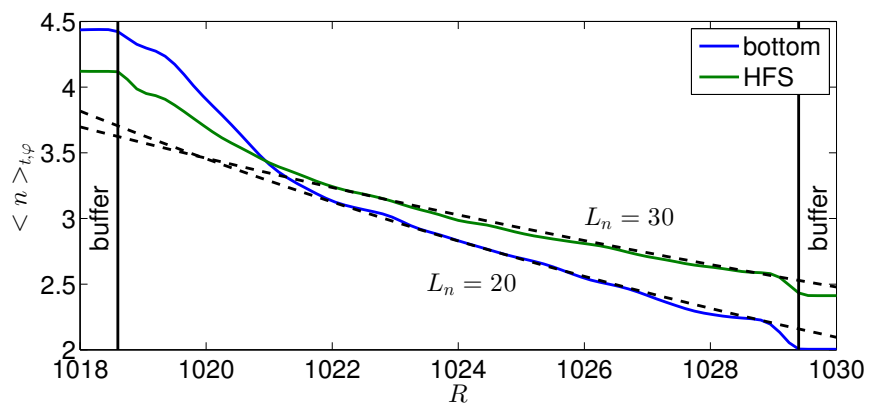

Figure 17: Density profile at outboard mid-plane averaged over time and toroidal angle for simulation with limiter at bottom and high field side. Black dashed lines are exponential fits to source free region with gradient lengths of $L_{n}=20$ respectively $L_{n}=30$.

on the low field side with its unfavourable curvature. Transport is stronger with the limiter at the high field side, which is supported by fig. 17 where the density profiles at outboard midplane are shown, yielding a gradient length of $L_{n}=20$ with limiter at bottom and $L_{n}=30$ with limiter at high field side.

Depending on the position of the limiter poloidal asymmetries of the average parallel velocity are observed in experiment [49]. A theoretical investigation revealed equilibrium $\mathrm{E} \times \mathrm{B}$ flows, due to the presence of the sheath, and pressure poloidal asymmetries as source with an analytic estimate $[50,51]$ (here: $\sigma_{\theta}=1$ and $\sigma_{\varphi}=1$ ) for the average Mach number $M:=-\left\langle u_{\|}^{i}\right\rangle_{t, \varphi}$ :

$$
\begin{aligned}
M\left(\rho^{*}, \theta^{*}\right)= & M_{s} e^{-\rho^{*} / l}+\left(M_{s h}+M_{a}\right)\left(1-e^{-\rho^{*} / l}\right)-2 \theta^{*}+ \\
& 4\left[\left(M_{s h}+M_{a}-M_{s}\right) e^{-\rho^{*} / l}-M_{a}\right] \theta^{*^{2}}
\end{aligned}
$$

with $\theta^{*}$ a poloidal coordinate ranging from -0.5 to 0.5 between the two limiter plates, and $\rho^{*}$ a flux surface label with $\rho^{*}=0$ at the separatrix. In practice we set $\rho^{*}=0$ at the location where the source free region starts. $M_{s}=M(0,0)$ is a reference Mach number and $M_{a}=(\delta n+\delta T) / 2$ is due to the pressure poloidal asymmetry, where $\delta n, \delta T$ is the density respectively temperature difference between the two limiter plates. For a comparison to our isothermal model we neglect $\delta T$ and $M_{s h}$, which represents an effect of the equilibrium electric field caused by the sheath and estimate the gradient length as $l \sim L_{n}$. The average Mach number obtained from GRILLIX and according to
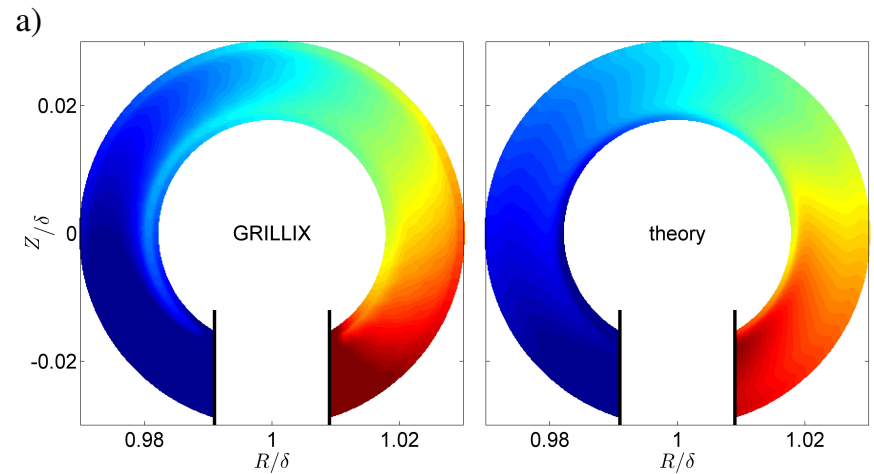

b)
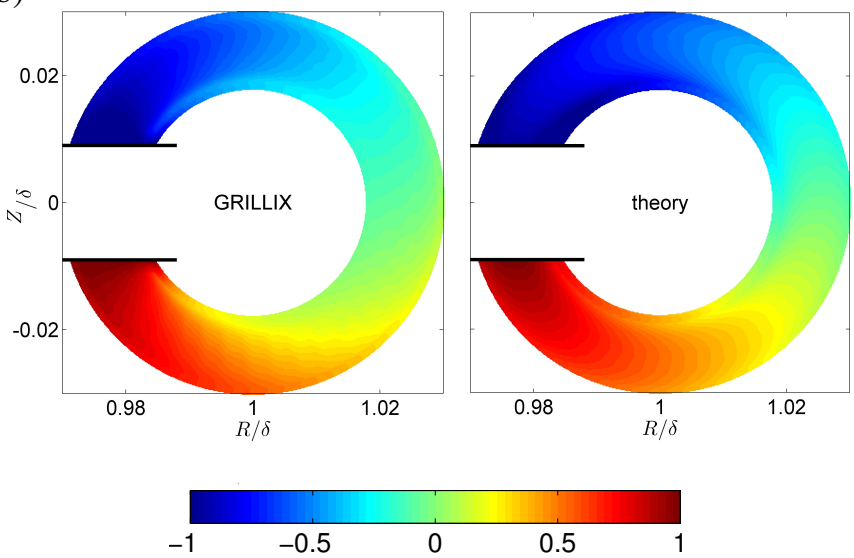

Figure 18: Average Mach number obtained from simulation with GRILLIX (left) and from theoretical estimate (right) according to equation (51). a) Limiter at bottom, b) limiter at high field side.

formula (51) is shown in fig. 18. Given the fact that formula (51) is only an estimate, the agreement is good as the poloidal asymmetry is reproduced correctly with GRILLIX.

In order to extract information along the parallel direction from GRILLIX simulations the characteristics of a magnetic field line $(R(\bar{\varphi}), Z(\bar{\varphi}), \bar{\varphi}))$ and its length $x_{\|}(\bar{\varphi})$ are computed via field line tracing (The non-cyclic toroidal angle is used here, i.e. $\varphi=\bar{\varphi} \bmod 2 \pi)$. Values of some quantity at intersection points of the magnetic field line with poloidal planes, i.e. $u\left(R\left(\bar{\varphi}_{k}\right), Z\left(\bar{\varphi}_{k}\right), \varphi_{k}\right)$, are obtained via interpolation. The density, parallel velocity and parallel current for three sample magnetic field lines are shown in fig. 19. There are no noticeable numerical corrugations along the parallel direction which indicates that field lines are globally resolved by the field line map, which connects only adjacent planes via interpolation. Moreover, the specified boundary conditions at the limiter plates (2226) are actually fulfilled.

\subsection{Divertor}

We consider again the geometry and magnetic configuration of section 5.1, but the simulation domain spans across the separatrix $\rho \in[0.88,1.06]$ with an $\mathrm{X}$-point at $\approx(0.85,-0.38)$. There is a horizontal divertor at $Z=-0.42$, and the private flux region is covered until $\rho=0.99$. 


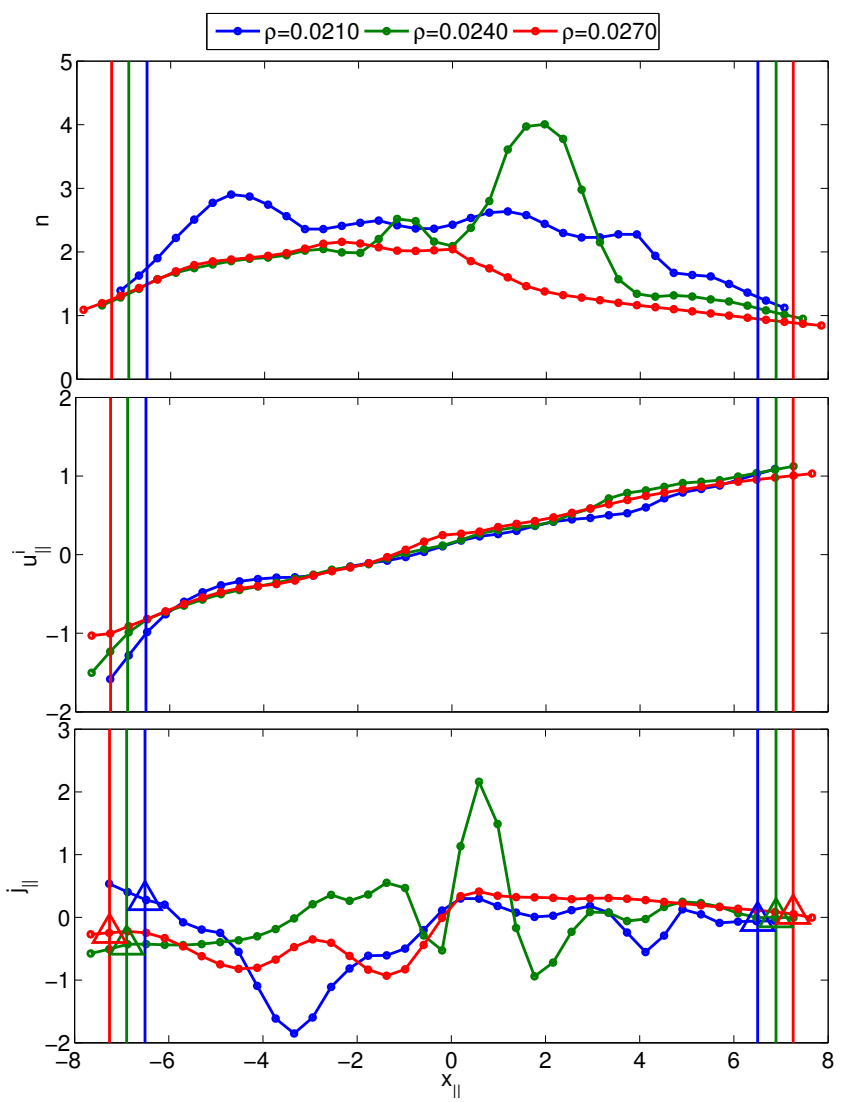

Figure 19: Density (top), parallel velocity (center) and parallel current (bottom) plotted along sample field lines for limiter simulation at $t=18$ with limiter at bottom. $x_{\|}$is coordinate along field line with $x_{\|}=0$ halfway between limiter plates. Three field lines running at different radial positions are shown and solid vertical lines mark intersection with limiter plates. The values of $\pm n \phi$ at the limiter plates are also marked in the bottom plot with triangles showing that the boundary condition (23) for the parallel current is actually fulfilled.
Before starting a turbulence simulation we established first a more suitable initial state via running GRILLIX as a transport code. Starting from low background density we ran GRILLIX without $E \times B$ advection and large nominal perpendicular diffusion $\left(N=2, v_{n}=v_{u}=v_{\Omega}=100\right)$ with a larger time step of $d t=1 \cdot 10^{-3}$ until a steady state was achieved. After this we switched on $E \times B$ advection and switched to hyper-diffusion $\left(N=2, v_{n}=v_{u}=v_{\Omega}=10\right)$. Other parameters were taken over from 5.1 except that we reduced again $\sigma=5$ in order to save computational costs and that we chose a higher toroidal resolution of $\Delta \varphi=2 \pi / 32$ with decreased parallel diffusion coefficients $\mu_{n}=\mu_{u}=0.04$.

A snapshot of the density in the saturated state is plotted in fig. 20. Whereas the high field side is rather quiescent with a jump in the density across the separatrix, the low field side exhibits strong fluctuations and swamps the separatrix. Also the effect of magnetic shear on structures is visible. Whereas they are rather isotropic at the unstable outboard side, they fan out towards the top and especially strongly towards the Xpoint, where they become subject to perpendicular dissipation $[23,52]$. There are also abrupt changes to the private flux region and the X-point becomes clearly visible. We emphasize here again that neither the grid nor the discrete operators are sensible to flux surfaces or the X-point. Each grid point is treated on the same footing, and the fact that the separatrix and the Xpoint become clearly visible in the simulation is a consequence of the field line map. Below the divertor line also the region of parallel ghost points is shown, which are essentially obtained from a linear extrapolation along magnetic field lines using information from the two neighbouring planes $\varphi_{k \pm 1}$ and $\varphi_{k \pm 2}$ (see equation B.6).

To give also an impression on the parallel structure we show in fig. 21 the parallel current on the flux surface $\rho=0.99$ close to the separatrix in dependency of the toroidal angle and the geometric poloidal angle. We want to note here once again that the complex courses of magnetic field lines become obvious in the texture although the computational grid is not aligned along magnetic field lines or flux surfaces. There is a disconnection along the parallel direction across the $\mathrm{X}$-point region, where the magnetic field lines run nearly purely toroidally. This is owed to strong dissipation in the vicinity of the X-point as structures are strongly fanned out by the local magnetic shear around the $\mathrm{X}$-point.

\section{Conclusions and Outlook}

The unique feature of GRILLIX is that it is based on the FCI approach, which exhibits a very high flexibility with respect to geometry. No special workaround for X-point(s) or magnetic axis is required, but every grid point is treated on the same footing. The numerical framework in GRILLIX consists of perpendicular operators which are easily discretised via standard finite difference methods within a set of Cartesian poloidal planes, and parallel operators which connect these planes via a field line map, i.e. field line tracing and interpolation. The discrete parallel divergence is thereby constructed from the discrete parallel 


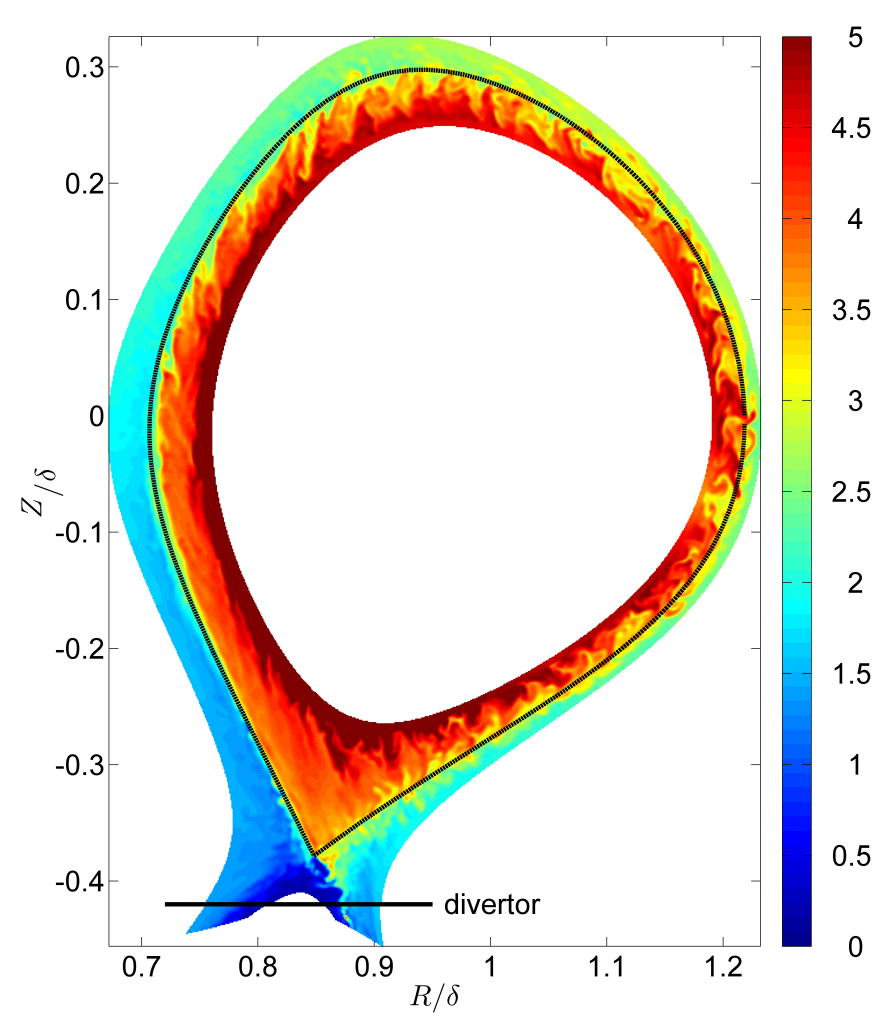

Figure 20: Snapshot of density of simulation with X-point. Separatrix is indicated with black dashed line and divertor plate with solid black line. Points below divertor at $Z=-0.42$ are ghost points.

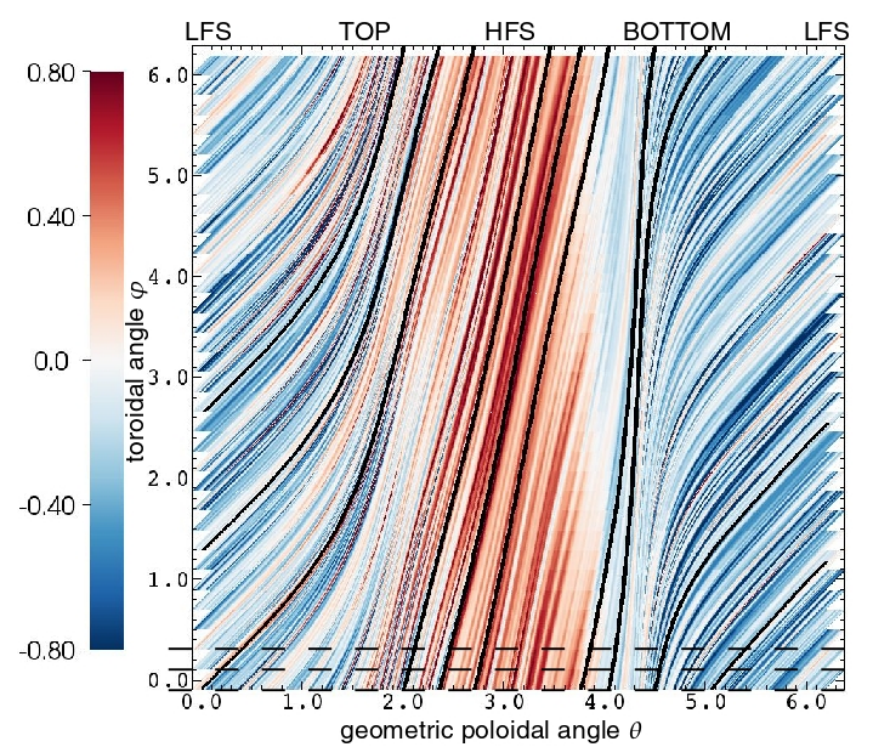

Figure 21: Snapshot of parallel current on flux surface $\rho=0.99$ as function of toroidal angle $\varphi$ and geometric poloidal angle $\theta=\arctan \left(\frac{Z / \delta}{R / \delta-1}\right)$. Low field side (LFS) corresponds to $\theta=0,2 \pi$, top to $\theta=\pi / 2$, high field side (HFS) to $\theta=\pi$ and bottom to $\theta=3 \pi / 2$. Part of a sample field line is indicated with solid black line and transitions between first planes with dashed black lines. gradient as its negative adjoint via application of the method of support operators in a toroidally staggered framework.

An isothermal electrostatic drift reduced version of the Braginskii model with Boussinesq approximation is employed in GRILLIX in order to model edge/SOL turbulence. An effective solution for the treatment of sheath boundary conditions, a subtle but important issue within the FCI approach, was presented. The implementation of the model was successfully verified with the method of manufactured solutions and a simple benchmark based on TORPEX blob experiments was performed.

Several examples showed the geometric flexibility of the code: The treatment of a magnetic axis within the FCI approach is trivial, and we showed the conservation of particles and the underlying energy theorem in a configuration with closed magnetic field lines. Pfirsch-Schlüter currents and the fluctuation level appeared thereby in correlation with the density profile. Pure SOL turbulence was investigated in toroidal limiter configuration. An example in diverted geometry showed that the structures align themselves automatically even to magnetic field lines with complicated courses. Although the computational grid of GRILLIX is neither aligned to field lines nor to flux surfaces, the separatrix, X-point and magnetic field lines become visible.

The verification tests and examples altogether are strong indications that the FCI approach in general and GRILLIX in particular are viable approaches in order to tackle modelling of edge/SOL turbulence in realistic diverted geometry. GRILLIX is still strongly capable of development, and the full drift reduced Braginskii model $[3,27]$ constitutes a rather complete model for edge/SOL turbulence, at least from the fluid point of view. Its further implementation into GRILLIX seems to be rather straight forward from now as discrete versions of all necessary operators are available. A geometric multigrid solver is already implemented in GRILLIX, which allows to relax the Boussinesq approximation, and inclusion of electron thermal effects has already been surveyed in GRILLIX within slab geometry [53]. Electromagnetic effects enter in the drift approximation as effective perpendicular dynamics. Also a gyrofluid model could be surveyed in order to treat finite larmor radius effects. Although GRILLIX is yet based on axisymmetry, there is no obvious reason why the developed methods could not easily be extended also to three-dimensional geometries [20], i.e. stellarators.

Finally, only obvious parallelisation and computational optimisations are currently applied in GRILLIX. A tedious but probably worthwhile task would be a controlled organisation of memory affinity in order to push OpenMP scalability. Moreover, a smart domain decomposition within poloidal planes would reduce communication and add another level of parallelisation.

\section{Acknowledgements}

The authors would like to thank F. Riva and F. Militello for providing data and analysing tools for the benchmark based on TORPEX blob experiments, B. Dudson for useful discussion on the Boussinesq approximation and energy theorem and J. Loizu 
for discussion on toroidal rotation in the SOL. This work has been carried out within the framework of the EUROfusion Consortium and has received funding from the Euratom research and training programme 2014-2018 under grant agreement No 633053. The views and opinions expressed herein do not necessarily reflect those of the European Commission. The support from the EUROfusion Researcher Fellowship programme under grant number AWP16-ERG-MPG/Stegmeir is gratefully acknowledged.

\section{Appendix A. Dissipation/sources in energy theorem}

The expressions for $D_{\epsilon_{\perp}}$ and $D_{\epsilon_{\|}}$in equations (15) and (16) are:

$$
\begin{aligned}
D_{\epsilon_{\perp}}= & -\phi \mathcal{D}_{\perp}(\Omega) \\
D_{\epsilon_{\|}}= & \frac{\epsilon_{\|}}{2 n}\left[\mathcal{D}_{\perp}(n)+\mathcal{D}_{\|}(n)-\mathcal{D}_{\perp}(\Omega)-S_{n}\right] \\
& +n u_{\|}^{i}\left[\mathcal{D}_{\perp}\left(u_{\|}^{i}\right)+\mathcal{D}_{\|}\left(u_{\|}^{i}\right)\right]
\end{aligned}
$$

\section{Appendix B. Full parallel boundary conditions}

The treatment of the full parallel boundary conditions (2226) according to the method touched upon in section 3.4 is presented here. Without loss of generality we restrict the discussion still to 2D within the setup of fig. 4 .

The expression for pure Neumann boundary condition is given in equation (41) and for the general case of a mixed type boundary condition:

$$
\left.\gamma \nabla_{\|} u\right|_{X}+\left.\beta u\right|_{X}=\alpha
$$

the value on ghost points is assigned according to:

$$
u_{A}=\frac{\alpha-\left(\gamma g_{B}+\beta f_{B}\right) u_{B}-\left(\gamma g_{C}+\beta f_{C}\right) u_{C}}{\gamma g_{A}+\beta f_{A}}
$$

with the coefficients:

$$
\begin{aligned}
f_{A}:=\frac{s_{B} s_{C}}{\left(s_{A}-s_{B}\right)\left(s_{A}-s_{C}\right)}, & g_{A}:=-f_{A} \frac{s_{B}+s_{C}}{s_{B} s_{C}}, \\
f_{B}:=-\frac{s_{A} s_{C}}{\left(s_{A}-s_{B}\right)\left(s_{B}-s_{C}\right)}, & g_{B}:=-f_{B} \frac{s_{A}+s_{C}}{s_{A} s_{C}}, \\
f_{C}:=\frac{s_{A} s_{B}}{\left(s_{A}-s_{C}\right)\left(s_{B}-s_{C}\right)}, & g_{C}:=-f_{C} \frac{s_{A}+s_{B}}{s_{A} s_{B}} .
\end{aligned}
$$

For a vanishing second parallel derivative $\left.\nabla_{\|}^{2} u\right|_{X}$ ghost points are set according to:

$$
u_{A}=\frac{s_{A}-s_{C}}{s_{B}-s_{C}} u_{B}+\frac{s_{B}-s_{A}}{s_{B}-s_{C}} u_{C},
$$

which covers the boundary conditions for the density (25) and vorticity (26) in the cases $K=1,2$.

The boundary condition (24) for the potential is of a mixed type with $\beta=-\left.\sigma^{-1} n\right|_{x}$ and $\alpha=\left.\nabla_{\|} \theta_{n}\right|_{X}$. Consistent with the Taylor expansion these values are obtained as

$$
\left.n\right|_{x}=f_{A} n_{A}+f_{B} n_{B}+f_{C} n_{C},\left.\quad \nabla_{\|} \theta_{n}\right|_{X}=g_{A} \theta_{n, A}+g_{B} \theta_{n, B}+g_{C} \theta_{n, C} .
$$

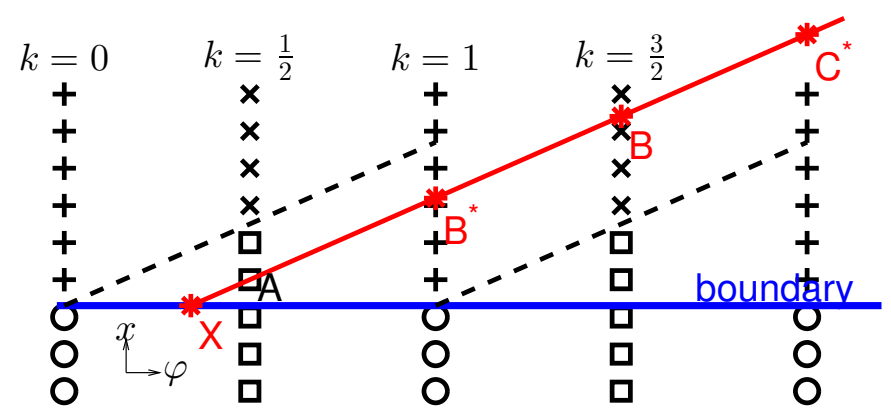

Figure B.22: Scheme for parallel boundary conditions within FCI on dual grid $G^{*}$. Grid points of $G$ are marked with '+' and grid points of $G^{*}$ with ' $\times$ '. Ghost points of $G$ are marked with 'o' and of $G^{*}$ with ' $\square$ ', which are below dashed line. Intersection (X) and map points (B,C) are defined as in fig. 4 but w.r.t. dual grid $G^{*}$. Additionally, map points with respect to grid $G$ are denoted with B* and $\mathrm{C}^{*}$.

The situation is slightly different for quantities defined on the dual grid $G^{*}$, i.e. for the parallel velocity and the parallel current. On $G^{*}$ also inner grid points which lie inside the domain are ghost points, for which the parallel gradient operator would involve ghost points from the canonical grid $G$. Otherwise the same methods and definitions, e.g. $\left(s_{A}, s_{B}, s_{C}\right)$, as for the canonical grid hold, but with respect to the dual grid (see fig. B.22).

For the Chodura boundary condition (22) $\left.u_{\|}^{i}\right|_{X}<-1$ we firstly extrapolate the parallel velocity to the intersection point according to:

$$
\tilde{u}_{\| X}^{i}=\frac{s_{C}}{s_{C}-s_{B}} u_{\| B}^{i}-\frac{s_{B}}{s_{C}-s_{B}} u_{\| C}^{i}
$$

and values at ghost points are then set according to equation (B.2) with $\gamma=0, \beta=1$ and $\alpha=\min \left(\tilde{u}_{\| X}^{i},-1\right)$.

Finally, the boundary condition (23) for the parallel current follows also according to equation (B.2) with $\gamma=0, \beta=1$ and $\alpha=-\left.n \phi\right|_{X}$. However, care must be taken as $j_{\|}$is defined on the dual grid $G^{*}$, but $n$ and $\phi$ on the grid $G$. One could beforehand map $n$ and $\phi$ to the staggered grid, which however would involve an additional interpolation. Instead we obtain the value for $\left.n\right|_{X}$ and $\left.\phi\right|_{X}$ from the map points $\mathrm{B}^{*}$ and $\mathrm{C}^{*}$ in fig. B.22 as:

$$
\left.n\right|_{X}=\frac{s_{C^{*}}}{s_{C^{*}}-s_{B^{*}}} n_{B^{*}}-\frac{s_{B^{*}}}{s_{C^{*}}-s_{B^{*}}} n_{B^{*}}
$$

with $s_{B^{*}}$ and $s_{C^{*}}$ the distances from the intersection point to the map points $\mathrm{B}^{*}$ and $\mathrm{C}^{*}$.

\section{Appendix C. Flux surface average in FCI}

Flux surface averages $\langle u\rangle_{\psi}$ have to be performed in GRILLIX for the source term and diagnostics purposes. As the grid is in general not aligned with flux surfaces in the FCI approach its computation requires some clarification.

In a flux aligned coordinate system $(\rho, \theta, \varphi)$ with $\rho$ some flux surface label and $\theta$ the geometric poloidal angle, the flux sur- 
face average is defined as [13]:

$$
\langle u\rangle_{\psi}:=\frac{\int_{0}^{2 \pi} \int_{0}^{2 \pi} d \theta d \varphi \sqrt{g} u}{\int_{0}^{2 \pi} \int_{0}^{2 \pi} d \theta d \varphi \sqrt{g}},
$$

with $\sqrt{g}$ the Jacobian, which can be computed directly via:

$$
\begin{aligned}
g^{-1}= & \frac{1}{(R / \delta)^{2}} \frac{1}{(R / \delta-1)^{2}+(Z / \delta)^{2}}\left[\left(\partial_{R} \rho / \delta\right)^{2}+\left(\partial_{Z} \rho / \delta\right)^{2}\right] \\
& -\frac{1}{(R / \delta)^{2}}\left[\frac{(R / \delta-1)\left(\partial_{Z} \rho / \delta\right)-(Z / \delta)\left(\partial_{R} \rho / \delta\right)}{(R / \delta-1)^{2}+(Z / \delta)^{2}}\right]^{2}
\end{aligned}
$$

On the discrete level we introduce a flux surface aligned grid with similar resolution as the cylindric grid, where the mapping $u\left(R_{i}, \varphi_{k}, Z_{j}\right) \rightarrow u\left(\rho_{m}, \theta_{n}, \varphi_{k}\right)$ can easily be performed with the available interpolation routines. The angle integrals are simply replaced by sums, i.e. $\int_{0}^{2 \pi} d \theta \rightarrow \sum_{n} \Delta \theta$ and $\int_{0}^{2 \pi} d \varphi \rightarrow \sum_{k} \Delta \varphi$, and the discrete flux surface operator is a sparse matrix $\mathbf{F}$ of dimension $n_{\rho} \times n_{c y l}$ with $n_{\rho}$ the number of flux surfaces of the flux aligned grid and $n_{c y l}$ the number of grid points of the cylindric grid. Finally, a 1D interpolation with the available $\langle u\rangle_{\psi_{m}}=(\mathbf{F u})_{m}$ is performed in order to obtain flux surface averages at arbitrary $\rho$.

[1] F. Hariri, M. Ottaviani, Comput. Phys. Commun. 184 (2013) 2419.

[2] A. Stegmeir, D. Coster, O. Maj, K. Hallatschek, K. Lackner, Comput. Phys. Commun. 198 (2016) 139.

[3] A. Zeiler, J. F. Drake, B. Rogers, Phys. Plasmas 4 (1997) 2134.

[4] F. Riva, C. Colin, J. Denis, L. Easy, I. Furno, J. Madsen, F. Militello, V. Naulin, A. H. Nielsen, J. M. B. Olsen, J. T. Omotani, J. J. Rasmussen, P. Ricci, E. Serre, P. Tamain, C. Theiler, Plasma Phys. Contr. F. 58 (2016) 044005.

[5] P. C. Stangeby, G. M. McCracken, Nucl. Fusion 30 (1990) 1225.

[6] M. R. O'Brian, D. C. Robinson, Tokamak experiments, in: R. O. Dendy (Ed.), Plasma Physics: an Indroductory Course, Cambridge University Press, Cambridge, 1993.

[7] Work Plan for the Implementation of the Fusion Roadmap in 2014-2018.

[8] J. J. Rasmussen, A. H. Nielsen, J. Madsen, V. Naulin, G. S. Xu, Plasma Phys. Contr. F. 58 (2016) 14031.

[9] P. Ricci, F. D. Halpern, S. Jolliet, J. Loizu, A. Mosetto, A. Fasoli, I. Furno, C. Theiler, Plasma Phys. Contr. F. 54 (2012) 124047.

[10] F. D. Halpern, P. Ricci, S. Jolliet, J. Loizu, J. Morales, A. Mosetto, F. Musil, F. Riva, T. M. Tran, C. Wersal, J. Comput. Phys. 315 (2016) 388.

[11] B. D. Dudson, J. Leddy, Plasma Phys. Contr. F. 59 (2017) 054010.

[12] P. Tamain, H. Bufferand, G. Ciraolo, C. Colin, D. Galassi, P. Ghendrih, F. Schwander, E. Serre, J. Comput. Phys. 321 (2016) 606.

[13] W. D. D'haeseleer, W. N. G. Hitchon, J. D. Callen, J. L. Sohet, Flux Coordinates and Magnetic Field Structure, Springer Series in Computational Physics, Springer, 1990.

[14] M. Ottaviani, Phys. Lett. A 375 (2011) 1677.

[15] F. Hariri, P. Hill, M. Ottaviani, Y. Sarazin, Phys. Plasmas 21 (2014) 082509.

[16] F. Hariri, P. Hill, M. Ottaviani, Y. Sarazin, Plasma Phys. Control. Fusion 57 (2015) 054001.

[17] A. Stegmeir, D. Coster, O. Maj, K. Lackner, Contrib. Plasm. Phys. 54 (2014) 549

[18] A. Stegmeir, O. Maj, D. Coster, K. Lackner, M. Held, M. Wiesenberger, Comput. Phys. Commun. 213 (2017) 111.

[19] M. Held, M. Wiesenberger, A. Stegmeir, Comput. Phys. Commun. 199 (2016) 29.
[20] P. Hill, B. Shanahan, B. Dudson, Comput. Phys. Commun 213 (2017) 9.

[21] F. Hariri, Fenicia: A generic plasma simulation code using a fluxindependent field-aligned coordinate approach, Ph.D. thesis, AixMarseille University (2013).

[22] A. Stegmeir, Grillix: A 3d turbulence code for magnetic fusion devices based on a field line map, Ph.D. thesis, Technical University of Munich, iPP report 5/135 (2015)

[23] A. Stegmeir, D. Coster, O. Maj, A. Ross, K. Lackner, 26th IAEA Fusion Energy Conference (2016) TH/P6-6.

[24] S. I. Braginskii, Transport processes in a plasma, in: A. M. A. Leontovich (Ed.), Reviews of Plasma Physics, Vol. 1, Consultants Bureau, 1965.

[25] K. Salari, P. Knupp, Code verification by the method of manufactured solutions, Sandia National Laboratories, Sandia Report SAND2000-1444 (2000).

[26] A. J. Wootton, B. A. Carreras, H. Matsumoto, K. McGuire, W. A. Peebles, C. P. Ritz, P. W. Terry, S. J. Zweben, Phys. Fluids. B 2 (1990) 2879.

[27] B. Scott, Low frequency fluid drift turbulence in magnetised plasmas, Habilitation treatise, Heinrich-Heine University Düsseldorf (2000).

[28] B. Dudson, Hermes model, https://github.com/boutproject/hermes/tree/master/doc/he manual.tex (2016).

[29] R. Chodura, Phys. Fluids. 25 (1982) 1628.

[30] P. C. Stangeby, The Plasma Boundary of Magnetic Fusion Devices, Plasma Physics Series, Institute of Physics Publishing, 2000.

[31] J. Loizu, P. Ricci, F. D. Halpern, S. Jolliet, Phys. Plasmas 19 (2012) 122307.

[32] M. Shashkov, S. Steinberg, J. Comput. Phys. 118 (1995) 131.

[33] M. Shashkov, Conservative Finite-Difference Methods on General Grids, CRC Press, 1996.

[34] A. Arakawa, J. Comput. Phys. 135 (1997) 103.

[35] G. E. Karniadakis, M. Israeli, S. A. Orszag, J. Comput. Phys. 97 (1991) 414.

[36] B. D. Scott, J. Comput. Phys. 78 (1988) 114.

[37] W. Hackbusch, Multi-Grid Methods and Applications, Springer-Verlag, 1985.

[38] A. Coco, G. Russo, J. Comput. Phys. 241 (2013) 464

[39] E. Hairer, S. P. Norsett, G. Wanner, Solving ordinary Differential Equations I. Nonstiff Problems, 2nd Edition, Springer Series in Computational Mathematics, Springer-Verlag, 1993.

[40] J. Williams, Modern Fortran Implementation of the DOP853 ODE Solver, https://github.com/jacobwilliams/dop853 (2016).

[41] W. H. Press, S. A. Teukolsky, W. T. Vetterling, B. P. Flannery, Numerical Recipes, 3rd Edition, Cambridge University Press, 2007.

[42] V. Frayssè, L. Giraud, S. Gratton, J. Langou, A set of GMRES routines for real and complex arithmetics on high performance computers, Cerfacs, Technical Report TR/PA/03/3 (2003).

[43] F. Riva, P. Ricci, F. D. Halpern, S. Jolliet, J. Loizu, A. Mosetto, Phys. Plasmas 21 (2014) 062301.

[44] B. D. Dudson, J. Madsen, J. Omotani, P. Hill, L. Easy, M. Loiten, Phys. Plasmas 23 (2016) 062303.

[45] I. Furno, F. Avino, A. Bovet, A. Diallo, A. Fasoli, K. Gustafson, D. Iraji, B. Labit, J. Loizu, S. H. Müller, G. Plyushchev, M. Podestà, F. M. Poli, P. Ricci, C. Theiler, J. Plasma Physics 81 (2015) 345810301.

[46] L. Easy, F. Militello, J. Omotani, B. Dudson, E. Havlíčková, P. Tamain, V. Naulin, A. H. Nielsen, Phys. Plasmas 21 (2014) 122515.

[47] P. J. M. Carthy, Phys. Plasmas 6 (1999) 3554.

[48] A. Dinklage, T. Klinger, G. Marx, L. Schweikhard (Eds.), Plasma Physics Confinement, transport and collective effects, Lecture notes in physics, Springer, 2005.

[49] J. P. Gunn, C. Boucher, M. Dionne, I. Ďuran, V. Fuchs, T. Loarer, I. Nanobashvili, R. Pánek, J.-Y. Pascal, F. Saint-Laurent, J. Stöckel, T. V. Rompuy, R. Zagórski, J. Adámek, J. Bucalossi, R. Dejarnac, P. Devynck, P. Hertout, M. Hron, G. Lebrun, P. Moreau, F. Rimini, A. Sarkissian, G. V. Ooost, J. Nucl. Mater. 363-365 (2007) 485.

[50] J. Loizu, P. Ricci, F. D. Halpern, S. Jolliet, A. Mosetto, Nucl. Fusion 54 (2014) 083033

[51] J. Loizu, P. Ricci, F. D. Halpern, S. Jolliet, A. Mosetto, Phys. Plasmas 21 (2014) 062309.

[52] D. Farina, R. Pozzoli, D. D. Ryutov, Nucl. Fusion 33 (1993) 1315.

[53] A. Ross, A. Stegmeir, D. Coster, Non-Boussinesq turbulence studies in the scrape-off layer, 16th International Workshop on Plasma Edge Theory in Fusion Devices, 27-29 Sep. 2017, Marseille, France (2017). 